\title{
On the Convergence of Gromov-Witten Potentials and Givental's Formula
}

\author{
Tom CoATES \& Hiroshi Iritani
}

\begin{abstract}
Let $X$ be a smooth projective variety. The Gromov-Witten potentials of $X$ are generating functions for the Gromov-Witten invariants of $X$ : they are formal power series, sometimes in infinitely many variables, with Taylor coefficients given by Gromov-Witten invariants of $X$. It is natural to ask whether these formal power series converge. In this paper we describe and analyze various notions of convergence for Gromov-Witten potentials. Using results of Givental and Teleman, we show that if the quantum cohomology of $X$ is analytic and generically semisimple, then the genus- $g$ Gromov-Witten potential of $X$ converges for all $g$. We deduce convergence results for the all-genus Gromov-Witten potentials of compact toric varieties, complete flag varieties, and certain noncompact toric varieties.
\end{abstract}

\section{Introduction}

Let $X$ be a smooth projective variety. The total descendant potential of $X$ is a generating function for the Gromov-Witten invariants of $X$. It is a formal power series $\mathcal{Z}_{X}$ in $\hbar, \hbar^{-1}$, and infinitely many variables $t_{k}^{\alpha}, 0 \leq \alpha \leq N, 0 \leq k<\infty$, with Taylor coefficients given by Gromov-Witten invariants of $X$. Here $t_{0}, t_{1}, t_{2}, \ldots$ is an infinite sequence of cohomology classes on $X, t_{k}=t_{k}^{0} \phi_{0}+\cdots+t_{k}^{N} \phi_{N}$ is the expansion of $t_{k}$ in terms of a basis $\left\{\phi_{\alpha}\right\}$ for $H^{\bullet}(X)$, and

$$
\mathcal{Z}_{X}=\exp \left(\sum_{g \geq 0} \hbar^{g-1} \mathcal{F}_{X}^{g}\right)
$$

where $\mathcal{F}_{X}^{g}$ is a generating function for genus- $g$ Gromov-Witten invariants. It is known that $\mathcal{Z}_{X}$ does not converge ${ }^{1}$ as a series in $\hbar$ and $\hbar^{-1}$, but it is natural to ask whether the formal power series $\mathcal{F}_{X}^{g}$ converge. This question is particularly relevant in light of work by Ruan and his collaborators on Gromov-Witten theory and birational geometry. If $X \rightarrow Y$ is a crepant birational map between smooth projective varieties (or orbifolds), then, very roughly speaking, the total descen-

Received December 3, 2014. Revision received June 3, 2015.

This research is supported by TC's Royal Society University Research Fellowship, ERC Starting Investigator Grant number 240123, the Leverhulme Trust, and Grant-in-Aid for Scientific Research (S) 23224002 and Grant-in-Aid for Young Scientists (B) 22740042.

${ }^{1} \mathcal{Z}_{X}$ should be regarded as an asymptotic expansion in $\hbar$. 
dant potentials $\mathcal{Z}_{X}$ and $\mathcal{Z}_{Y}$ are conjectured to be related by analytic continuation in the parameters $t_{i}^{\alpha}$. Implicit here, then, is the conjecture that the power series defining $\mathcal{F}_{X}^{g}$ and $\mathcal{F}_{Y}^{g}$ converge.

There are several different notions of convergence for a power series in infinitely many variables. We say that the total descendant potential $\mathcal{Z}_{X}$ is NFconvergent (see Definition 7.5) if each genus- $g$ descendant potential $\mathcal{F}_{X}^{g}$ converges on an infinite-dimensional polydisc of the form shown in equation (30) below. This implies that each $\mathcal{F}^{g}$ defines a holomorphic function on a neighborhood of zero in an appropriate nuclear Fréchet space; see Remark 7.6. The main result of this paper (Theorem 1.1) is that if $X$ is a projective variety such that the quantum cohomology of $X$ is analytic and generically semisimple, then the total descendant potential $\mathcal{Z}_{X}$ is NF-convergent.

The quantum cohomology of $X$ is a family of algebra structures on $H^{\bullet}(X)$ parameterized by a point $t \in H^{\bullet}(X)$. The structure constants of the quantum cohomology algebra are formal power series in $t^{\alpha}, 0 \leq \alpha \leq N$, where $t=$ $t^{0} \phi_{0}+\cdots+t^{N} \phi_{N}$ is the expansion of $t$ with respect to a basis $\left\{\phi_{\alpha}\right\}$ for $H^{\bullet}(X)$, with Taylor coefficients given by genus-zero Gromov-Witten invariants of $X$; see Section 2.3. We consider three conditions on the Gromov-Witten invariants of $X$ :

Formal Semisimplicity: (see equation (24)) roughly speaking, it states that the quantum cohomology algebra of $X$ is semisimple at the generic point of a formal neighborhood of the large-radius limit point;

Genus-Zero Convergence: (see equation (25)) roughly speaking, it states that the power series defining the quantum cohomology algebra converge to give analytic functions of $t^{0}, \ldots, t^{N}$; and

Analytic Semisimplicity: (see equation (26)) it asserts that the resulting analytic family of algebras is semisimple for generic $t \in H^{\bullet}(X)$.

Formal Semisimplicity and Genus-Zero Convergence together imply Analytic Semisimplicity, and Genus-Zero Convergence and Analytic Semisimplicity together imply Formal Semisimplicity.

THEOREM 1.1. Let X be a smooth projective variety that satisfies Formal Semisimplicity, Genus-Zero Convergence, and Analytic Semisimplicity. The total descendant potential $\mathcal{Z}_{X}$ is NF-convergent in the sense of Definition 7.5.

Theorem 1.1 is proved in Section 7. It has the following immediate consequences.

COROLlARY 1.2. Let $X$ be a compact toric variety or a complete flag variety. The total descendant potential $\mathcal{Z}_{X}$ is $N F$-convergent in the sense of Definition 7.5.

Proof. By Theorem 1.1 it suffices to show that $X$ satisfies Genus-Zero Convergence and Analytic Semisimplicity. If $X$ is a compact toric variety, then this follows from mirror symmetry $[17 ; 24 ; 25]$. If $X$ is a complete flag variety, then this follows from mirror symmetry $[16 ; 28]$, reconstruction theorems for logarithmic Frobenius manifolds [35] [25, Prop. 5.8], and the work of Kostant [31]. 
Theorem 1.1 also implies the NF-convergence of the total descendant potential $\mathcal{Z}_{X}$ when $X$ is the total space of a direct sum of negative line bundles over a compact toric variety. This includes the case where $X=K_{Y}$ is the total space of the canonical line bundle over a compact Fano toric variety $Y$.

Corollary 1.3. Let $Y$ be a compact toric variety, and let $X$ be the total space of a direct sum $E=\bigoplus_{j=1}^{j=r} E_{j}$ of line bundles $E_{j}$ over $Y$ such that $c_{1}\left(E_{j}\right) \cdot d<$ 0 whenever $d$ is the degree of a holomorphic curve in $Y$. The total descendant potential $\mathcal{Z}_{X}$ is $N F$-convergent in the sense of Definition 7.5.

Corollary 1.3 is proved in Section 9.

We deduce Theorem 1.1 from a more fundamental result, Theorem 1.4, concerning the convergence of the total ancestor potential $\mathcal{A}_{X}$. The total ancestor potential is a generating function for ancestor Gromov-Witten invariants (see equations (8)-(10)). We say that the total ancestor potential $\mathcal{A}_{X}$ is NF-convergent if it is convergent on an infinite-dimensional polydisc as before (see equation (29)). We consider also a stronger notion of convergence for $\mathcal{A}_{X}$ (see Definition 3.13), requiring that in terms of the dilaton-shifted coordinates introduced in Section 2.6, we have

$$
\mathcal{A}_{X}=\exp \left(\sum_{g=0}^{\infty} \hbar^{g-1} \overline{\mathcal{F}}_{t}^{g}\right),
$$

where

$$
\overline{\mathcal{F}}_{t}^{g}=\sum_{n: 3 g-3+n \geq 0} \frac{1}{n !} \sum_{\substack{I: I=\left(i_{1}, \ldots, i_{n}\right) \\ i_{j} \neq 1 \text { for all } j \\ i_{1}+\cdots+i_{n} \leq 3 g-3+n}} \sum_{A=\left(\alpha_{1}, \ldots, \alpha_{n}\right)} C_{I, A}^{(g)}\left(t, q_{1}\right) q_{i_{1}}^{\alpha_{1}} \cdots q_{i_{n}}^{\alpha_{n}}
$$

for some analytic functions $C_{I, A}^{(g)}\left(t, q_{1}\right)$ of $\left(t, q_{1}\right)$ that are rational in $q_{1}$ unless $(g, n)=(1,0)$ (see (16)). Convergence in this sense implies that the genus- $g$ ancestor potential $\overline{\mathcal{F}}_{t}^{g}$ is a formal power series in $q_{0}^{\alpha}$ with coefficients that depend polynomially on $q_{i}^{\alpha}, i>1$, and holomorphically on $t$ and $q_{1}^{\alpha}$; furthermore, Givental's tameness condition [20] holds.

THeOREM 1.4. Let X be a smooth projective variety that satisfies Formal Semisimplicity, Genus-Zero Convergence, and Analytic Semisimplicity. The total ancestor potential $\mathcal{A}_{X}$ is NF-convergent in the sense of Definition 7.1 and is convergent in the sense of Definition 3.13.

The rationality condition on $\mathcal{A}_{X}$ and the definition of the ancestor Fock space in which $\mathcal{A}_{X}$ lies were developed as part of a joint project with Hsian-Hua Tseng. We would like to thank him for allowing us to present the Fock space formulation in this paper. 
We now discuss the work of Givental [18; 19] and Teleman [36] on highergenus potentials for target spaces with semisimple quantum cohomology. This is an essential ingredient in the proof of Theorem 1.4. Motivated by an ingenious localization computation in torus-equivariant Gromov-Witten theory, Givental conjectured a formula that determines higher-genus Gromov-Witten potentials in terms of genus-zero data alone. His formula makes sense for any semisimple Frobenius manifold. In order to distinguish it from the geometric Gromov-Witten potential, we call the potential associated to a Frobenius manifold via Givental's formula the abstract potential.

Teleman [36] has shown that for any semisimple cohomological field theory (CohFT) satisfying a homogeneity condition and a flat vacuum condition, the potential associated to the CohFT coincides with Givental's abstract potential. Since Gromov-Witten theory defines a CohFT satisfying the homogeneity and flat vacuum conditions, Teleman's theorem applies to Gromov-Witten theory whenever the genus-zero part (quantum cohomology) is semisimple. There is a subtlety here. Quantum cohomology is a formal family of algebras parameterized by Novikov variables $Q_{i}$ and cohomology parameters $t^{0}, \ldots, t^{N}$ as before, and its convergence is not known in general. At the origin $Q_{i}=t^{j}=0$, the quantum cohomology coincides with the classical cohomology ring and so is semisimple only when the target $X$ is a point. At first sight, then, it appears that to apply Teleman's theorem, we need to find a semisimple point in the parameter space where all higher-genus Gromov-Witten potentials converge. (To prove this directly is beyond the reach of current methods in all but the very simplest examples.) In fact, this is not the case: as Teleman points out in [36, Example 1.6], his theorem applies whenever the quantum cohomology "at the generic point" in the formal neighborhood of the origin is semisimple. Thus, Givental's abstract potential can be defined and coincides with the geometric Gromov-Witten potential under our assumption of Formal Semisimplicity (24). If in addition Genus-Zero Convergence holds, then it follows that the higher-genus Gromov-Witten potentials, which a priori are only formal power series, in fact, converge to give analytic functions.

We expand upon these points in the rest of the paper. In Section 2 we fix notation for Gromov-Witten invariants, generating functions, and quantum cohomology. In Section 3 we describe Givental's quantization formalism. We then discuss Givental's formula in the analytic setting (Section 4) and in the formal setting (Section 5) and explain how Givental's formula follows from Teleman's classification theorem (Section 6). Results about the NF convergence of ancestor and descendant potentials are stated in Section 7 and proved in Section 8. We conclude with the proof of Corollary 1.3 in Section 9.

\section{Preliminaries}

Let $X$ be a smooth projective variety, and let $H_{X}$ be the even part of $H^{\bullet}(X ; \mathbb{Q})$. 


\subsection{Gromov-Witten Invariants}

Let $X_{g, n, d}$ denote the moduli space of $n$-pointed genus- $g$ stable maps to $X$ of degree $d \in H_{2}(X ; \mathbb{Z})$. Write

$$
\left\langle a_{1} \psi_{1}^{i_{1}}, \ldots, a_{n} \psi_{n}^{i_{n}}\right\rangle_{g, n, d}^{X}=\int_{\left[X_{g, n, d}\right]^{\mathrm{vir}}} \prod_{k=1}^{k=n} \operatorname{ev}_{k}^{\star}\left(a_{k}\right) \cup \psi_{k}^{i_{k}},
$$

where $a_{1}, \ldots, a_{n} \in H_{X}, \mathrm{ev}_{k}: X_{g, n, d} \rightarrow X$ is the evaluation map at the $k$ th marked point, $\psi_{1}, \ldots, \psi_{n} \in H^{2}\left(X_{g, n, d} ; \mathbb{Q}\right)$ are the universal cotangent line classes, $i_{1}, \ldots, i_{n}$ are nonnegative integers, and the integral denotes cap product with the virtual fundamental class $[3 ; 32]$. The right-hand side of (1) is a rational number, called a Gromov-Witten invariant of $X$ (if $i_{k}=0$ for all $k$ ) or a gravitational descendant (if any of the $i_{k}$ are nonzero).

\subsection{Bases for Cohomology and Novikov Rings}

Fix bases $\phi_{0}, \ldots, \phi_{N}$ and $\phi^{0}, \ldots, \phi^{N}$ for $H_{X}$ such that:

- $\phi_{0}$ is the identity element of $H_{X}$,

- $\phi_{1}, \ldots, \phi_{r}$ is a nef $\mathbb{Z}$-basis for $H^{2}(X ; \mathbb{Z}) \subset H_{X}$,

- each $\phi_{i}$ is homogeneous,

- $\left(\phi_{i}\right)_{i=0}^{i=N}$ and $\left(\phi^{j}\right)_{j=0}^{j=N}$ are dual with respect to the Poincaré pairing.

Note that $r$ is the rank of $H_{2}(X)$. Define the Novikov ring $\Lambda=\mathbb{Q} \llbracket Q_{1}, \ldots, Q_{r} \rrbracket$ and, for $d \in H_{2}(X ; \mathbb{Z})$, write

$$
Q^{d}=Q_{1}^{d_{1}} \cdots Q_{r}^{d_{r}}
$$

where $d_{i}=d \cdot \phi_{i}$.

\subsection{Quantum Cohomology}

Let $t^{0}, \ldots, t^{N}$ be the coordinates on $H_{X}$ defined by the basis $\phi_{0}, \ldots, \phi_{N}$, so that $t \in H_{X}$ satisfies $t=t^{0} \phi_{0}+\cdots+t^{N} \phi_{N}$. Define the genus-zero Gromov-Witten potential $F_{X}^{0} \in \Lambda \llbracket t^{0}, \ldots, t^{N} \rrbracket$ by

$$
F_{X}^{0}=\sum_{d \in \mathrm{NE}(X)} \sum_{n=0}^{\infty} \frac{Q^{d}}{n !}\langle t, \ldots, t\rangle_{0, n, d}^{X},
$$

where the first sum is over the set $\mathrm{NE}(X)$ of degrees of effective curves in $X$. This is a generating function for genus-zero Gromov-Witten invariants. The quantum product $*$ is defined in terms of the third partial derivatives of $F_{X}^{0}$ :

$$
\phi_{\alpha} * \phi_{\beta}=\sum_{\gamma=0}^{\gamma=N} \frac{\partial^{3} F_{X}^{0}}{\partial t^{\alpha} \partial t^{\beta} \partial t^{\gamma}} \phi^{\gamma} .
$$

The product $*$ is bilinear over $\Lambda$ and defines a formal family of algebras on $H_{X} \otimes$ $\Lambda$ parameterized by $t^{0}, \ldots, t^{N}$. This is the quantum cohomology or big quantum cohomology of $X$. 
We have defined big quantum cohomology as a formal family of algebras, that is, in terms of the ring of formal power series $\mathbb{Q} \llbracket Q_{1}, \ldots, Q_{r} \rrbracket \llbracket t^{0}, \ldots, t^{N} \rrbracket$. In many cases however, the genus-zero Gromov-Witten potential $F_{X}^{0}$ converges to an analytic function. By this we mean the following. The divisor equation [29, Sect. 2.2.4] implies that

$$
F_{X}^{0} \in \mathbb{Q} \llbracket t^{0}, Q_{1} e^{t^{1}}, \ldots, Q_{r} e^{t^{r}}, t^{r+1}, t^{r+2}, \ldots, t^{N} \rrbracket,
$$

and it can be often shown, for example, by using mirror symmetry, that $F_{X}^{0}$ is the power series expansion of an analytic function:

$$
F_{X}^{0} \in \mathbb{Q}\left\{t^{0}, Q_{1} e^{t^{1}}, \ldots, Q_{r} e^{t^{r}}, t^{r+1}, t^{r+2}, \ldots, t^{N}\right\} .
$$

We can then set $Q_{1}=\cdots=Q_{r}=1$, obtaining an analytic function

$$
F_{X}^{0} \in \mathbb{Q}\left\{t^{0}, e^{t^{1}}, \ldots, e^{t^{r}}, t^{r+1}, t^{r+2}, \ldots, t^{N}\right\}
$$

of the variables $t^{0}, \ldots, t^{N}$ defined in a region

$$
\left\{\begin{array}{l}
\left|t^{i}\right|<\epsilon_{i}, \quad i=0 \text { or } r<i \leq N, \\
\Re t^{i} \ll 0, \quad 1 \leq i \leq r
\end{array}\right.
$$

where $\mathfrak{R} t^{i}$ means the real part of $t^{i}$. We refer to the limit point

$$
\begin{cases}t^{i}=0, & i=0 \text { or } r<i \leq N \\ \Re t^{i} \rightarrow-\infty, & 1 \leq i \leq r,\end{cases}
$$

as the large-radius limit point. When $F_{X}^{0}$ converges to an analytic function in the sense just described, the quantum product $*$ then defines a family of algebra structures on $H_{X}$ that depends analytically on parameters $t^{0}, \ldots, t^{N}$ in the neighborhood (4) of the large-radius limit point.

REMARK 2.1. In this paper we only consider the even part of the cohomology group, but this is not really a restriction. Hertling, Manin, and Teleman [23] proved that if the quantum cohomology of a smooth projective variety $X$ is semisimple, then $X$ has no odd cohomology and is of Hodge-Tate type: $H^{p, q}(X)=0$ for $p \neq q$.

\subsection{The Dubrovin Connection}

Consider $H_{X} \otimes \Lambda$ as a scheme over $\Lambda$ and let $\mathcal{M}$ be a formal neighborhood of the origin in $\mathcal{M}$. The Euler vector field $E$ on $\mathcal{M}$ is

$$
E=t^{0} \frac{\partial}{\partial t^{0}}+\sum_{i=1}^{r} \rho^{i} \frac{\partial}{\partial t^{i}}+\sum_{i=r+1}^{N}\left(1-\frac{1}{2} \operatorname{deg} \phi_{i}\right) t^{i} \frac{\partial}{\partial t^{i}},
$$

where $c_{1}(X)=\rho^{1} \phi_{1}+\cdots+\rho^{r} \phi_{r}$. The grading operator $\mu: H_{X} \rightarrow H_{X}$ is defined by

$$
\mu\left(\phi_{i}\right)=\left(\frac{1}{2} \operatorname{deg} \phi_{i}-\frac{1}{2} \operatorname{dim}_{\mathbb{C}} X\right) \phi_{i} .
$$


Let $\pi: \mathcal{M} \times \mathbb{A}^{1} \rightarrow \mathcal{M}$ denote projection to the first factor. The extended Dubrovin connection is a meromorphic flat connection $\nabla$ on $\pi^{\star} T \mathcal{M} \cong H_{X} \times\left(\mathcal{M} \times \mathbb{A}^{1}\right)$, defined by

$$
\begin{aligned}
& \nabla_{\partial / \partial t_{i}}=\frac{\partial}{\partial t_{i}}-\frac{1}{z}\left(\phi_{i} *\right), \quad 0 \leq i \leq N \\
& \nabla_{z \partial / \partial z}=z \frac{\partial}{\partial z}+\frac{1}{z}(E *)+\mu, \quad \text { where } z \text { is the coordinate on } \mathbb{A}^{1} .
\end{aligned}
$$

Together with the pairing on $T \mathcal{M}$ induced by the Poincare pairing, the Dubrovin connection equips $\mathcal{M}$ with the structure of a formal Frobenius manifold with extended structure connection [33].

If the genus-zero Gromov-Witten potential $F_{X}^{0}$ converges to an analytic function, as discussed in Section 2.3, then the extended Dubrovin connection with $Q_{1}=\cdots=Q_{r}=1$ depends analytically on $t$ in a neighborhood (4) of the largeradius limit point and defines an analytic Frobenius manifold with extended structure connection.

\subsection{Gromov-Witten Potentials}

We begin by defining the formal power series ring to which the Gromov-Witten potentials belong. The Novikov ring $\Lambda$ is topologized by regarding it as the completion of the polynomial ring $\mathbb{Q}\left[Q_{1}, \ldots, Q_{r}\right]$ with respect to the valuation $v$ such that $v\left(Q^{d}\right)=d \cdot \omega$, where $\omega$ is a Kähler class on $X$. We will need also certain related formal power series rings, shown in Table 1 . These are defined as the completions of polynomial rings, shown in the second column of Table 1, with respect to a valuation $v$ such that

$$
v\left(Q^{d}\right)=d \cdot \omega, \quad v\left(t^{\alpha}\right)=1, \quad v\left(t_{i}^{\alpha}\right)=i+1, \quad v\left(y_{j}^{\beta}\right)=j+1 .
$$

The valuation $v$ yields a "maximal" completion of the polynomial rings; for example, $\Lambda \llbracket \mathbf{t} \rrbracket$ contains an arbitrary infinite sum $\sum_{\mathbf{m}} a_{\mathbf{m}} \mathbf{t}^{\mathbf{m}}$ with $a_{\mathbf{m}} \in \Lambda$, where $\mathbf{t}^{\mathbf{m}}$ stands for a (finite) monomial in the variables $\left\{t_{i}^{\alpha}: 0 \leq i<\infty, 0 \leq \alpha \leq N\right\}$. For a ring $R$ equipped with nonnegative valuation $v$, we define

$$
R\left\{\hbar^{-1}, \hbar \rrbracket=\left\{\sum_{n=-\infty}^{n=\infty} a_{n} \hbar^{n}: a_{n} \in R, \lim _{n \rightarrow-\infty} v\left(a_{n}\right)=\infty\right\} .\right.
$$

Table 1 Formal Power Series Rings

\begin{tabular}{lc}
\hline \hline Completed Ring & Underlying Polynomial Ring \\
\hline$\Lambda$ & $\mathbb{Q}\left[Q_{1}, \ldots, Q_{r}\right]$ \\
$\Lambda \llbracket t \rrbracket$ & $\mathbb{Q}\left[Q_{1}, \ldots, Q_{r}\right]\left[t^{\alpha}: 0 \leq \alpha \leq N\right]$ \\
$\Lambda \llbracket \mathbf{t} \rrbracket$ & $\mathbb{Q}\left[Q_{1}, \ldots, Q_{r}\right]\left[t_{i}^{\alpha}: 0 \leq i<\infty, 0 \leq \alpha \leq N\right]$ \\
$\Lambda \llbracket \mathbf{y} \rrbracket \llbracket t \rrbracket$ & $\mathbb{Q}\left[Q_{1}, \ldots, Q_{r}\right]\left[y_{j}^{\beta}: 0 \leq j<\infty, 0 \leq \beta \leq N\right]\left[t^{\alpha}: 0 \leq \alpha \leq N\right]$ \\
\hline \hline
\end{tabular}


Let $\mathbf{t}=\left(t_{0}, t_{1}, t_{2}, \ldots\right)$ be an infinite sequence of elements of $H_{X}$ and write $t_{i}=t_{i}^{0} \phi_{0}+\cdots+t_{i}^{N} \phi_{N}$. Define the genus- $g$ descendant potential $\mathcal{F}_{X}^{g} \in \Lambda \llbracket \mathbf{t} \rrbracket$ by

$$
\mathcal{F}_{X}^{g}=\sum_{d \in \mathrm{NE}(X)} \sum_{n=0}^{\infty} \sum_{i_{1}=0}^{\infty} \cdots \sum_{i_{n}=0}^{\infty} \frac{Q^{d}}{n !}\left\langle t_{i_{1}} \psi_{1}^{i_{1}}, \ldots, t_{i_{n}} \psi_{n}^{i_{n}}\right\rangle_{g, n, d}^{X} .
$$

This is a generating function for genus- $g$ gravitational descendants. The total descendant potential $\mathcal{Z}_{X} \in \Lambda \llbracket \mathbf{t} \rrbracket\left\{\hbar^{-1}, \hbar \rrbracket\right.$ is

$$
\mathcal{Z}_{X}=\exp \left(\sum_{g=0}^{\infty} \hbar^{g-1} \mathcal{F}_{X}^{g}\right)
$$

This is a generating function for all gravitational descendants of $X$. Note that $\left.\mathcal{F}_{X}^{0}\right|_{Q=\mathbf{t}=0}=0$, and thus we have $v\left(\mathcal{F}_{X}^{0}\right)>0$ with respect to the valuation $v$; this implies that $\mathcal{Z}_{X}$ belongs to $\Lambda \llbracket \mathbf{t} \rrbracket\left\{\hbar^{-1}, \hbar \rrbracket\right.$.

Consider now the map $p_{m}: X_{g, m+n, d} \rightarrow \overline{\mathcal{M}}_{g, m}$ that forgets the map and the last $n$ marked points, and then stabilizes the resulting prestable curve. Write $\psi_{m \mid i} \in H^{2}\left(X_{g, n+m, d} ; \mathbb{Q}\right)$ for the pullback along $p_{m}$ of the $i$ th universal cotangent line class on $\overline{\mathcal{M}}_{g, m}$, and

$$
\begin{aligned}
& \left\langle a_{1} \bar{\psi}_{1}^{i_{1}}, \ldots, a_{m} \bar{\psi}^{i_{m}}: b_{1}, \ldots, b_{n}\right\rangle_{g, m+n, d}^{X} \\
& \quad=\int_{\left[X_{g, m+n, d}\right]^{\mathrm{vir}}} \prod_{k=1}^{k=m}\left(\operatorname{ev}_{k}^{\star}\left(a_{k}\right) \cup \psi_{m \mid k}^{i_{k}}\right) \cdot \prod_{l=m+1}^{l=m+n} \operatorname{ev}_{l}^{\star}\left(b_{l-m}\right),
\end{aligned}
$$

where $a_{1}, \ldots, a_{m} \in H_{X}, b_{1}, \ldots, b_{n} \in H_{X}$, and $i_{1}, \ldots, i_{m}$ are nonnegative integers.

As before, consider $t \in H_{X}$ with $t=t^{0} \phi_{0}+\cdots+t^{N} \phi_{N}$ and an infinite sequence $\mathbf{y}=\left(y_{0}, y_{1}, y_{2}, \ldots\right)$ of elements in $H_{X}$ with $y_{i}=y_{i}^{0} \phi_{0}+\cdots+y_{i}^{N} \phi_{N}$. The genus-g ancestor potential $\overline{\mathcal{F}}_{X}^{g} \in \Lambda \llbracket \mathbf{y} \rrbracket \llbracket t \rrbracket$ is defined by

$$
\overline{\mathcal{F}}_{X}^{g}=\sum_{d \in \operatorname{NE}(X)} \sum_{n=0}^{\infty} \sum_{m=0}^{\infty} \sum_{j_{1}=0}^{\infty} \ldots \sum_{j_{m}=0}^{\infty} \frac{Q^{d}}{n ! m !}\langle y_{j_{1}} \bar{\psi}_{1}^{j_{1}}, \ldots, y_{j_{m}} \bar{\psi}_{m}^{j_{m}}: \overbrace{t, \ldots, t}^{n}\rangle_{g, m+n, d}^{X},
$$

and the total ancestor potential $\mathcal{A}_{X} \in \Lambda \llbracket \mathbf{y} \rrbracket \llbracket t \rrbracket\left\{\hbar^{-1}, \hbar \rrbracket\right.$ is

$$
\mathcal{A}_{X}=\exp \left(\sum_{g=0}^{\infty} \hbar^{g-1} \overline{\mathcal{F}}_{X}^{g}\right) \text {. }
$$

We will often want to emphasize the dependence of the ancestor potentials on the variable $t$, writing $\overline{\mathcal{F}}_{t}^{g}$ for $\overline{\mathcal{F}}_{X}^{g}$ and $\mathcal{A}_{t}$ for $\mathcal{A}_{X}$. Note that the ancestor potentials (9) do not contain terms with $g=0$ and $m<3$ or with $g=1$ and $m=0$ since in these cases the space $\overline{\mathcal{M}}_{g, m}$ is empty and so the map $p_{m}: X_{g, m+n, d} \rightarrow \overline{\mathcal{M}}_{g, m}$ is not defined. 


\subsection{Dilaton Shift}

Consider now another sequence $\mathbf{q}=\left(q_{0}, q_{1}, q_{2}, \ldots\right)$ with $q_{i} \in H_{X}$, and write $q_{i}=$ $q_{i}^{0} \phi_{0}+\cdots+q_{i}^{N} \phi_{N}$. We regard $\left\{q_{i}^{\alpha}: 0 \leq i<\infty, 0 \leq \alpha \leq N\right\}$ as a coordinate system on $H_{X} \llbracket z \rrbracket$ by writing a general point in $H_{X} \llbracket z \rrbracket$ as $\mathbf{q}(z)=\sum_{i=0}^{\infty} q_{i} z^{i}$. The dilaton shift is an identification between $\mathbf{q}=\left(q_{0}, q_{1}, q_{2}, \ldots\right)$ and the arguments $\mathbf{t}=\left(t_{0}, t_{1}, t_{2}, \ldots\right), \mathbf{y}=\left(y_{0}, y_{1}, y_{2}, \ldots\right)$ of the descendant and ancestor potentials:

$$
q_{i}^{\alpha}=\left\{\begin{array}{ll}
t_{1}^{0}-1 & \text { if }(i, \alpha)=(1,0), \\
t_{i}^{\alpha} & \text { otherwise, }
\end{array} \quad q_{i}^{\alpha}= \begin{cases}y_{1}^{0}-1 & \text { if }(i, \alpha)=(1,0), \\
y_{i}^{\alpha} & \text { otherwise }\end{cases}\right.
$$

Setting $\mathbf{t}(z)=\sum_{i=0}^{\infty} t_{i} z^{i}$ and $\mathbf{y}(z)=\sum_{i=0}^{\infty} y_{i} z^{i}$, the dilaton shift becomes the equalities:

$$
\mathbf{q}(z)=\mathbf{t}(z)-\phi_{0} z, \quad \mathbf{q}(z)=\mathbf{y}(z)-\phi_{0} z
$$

In this way we regard the descendant potential $\mathcal{F}_{X}^{g}$ as a function on the formal neighborhood of the point $-\phi_{0} z \in H_{X} \llbracket z \rrbracket$. The dilaton shift for the ancestor potential is discussed in Example 3.7.

\subsection{The Orbifold Case}

The results in this paper are all valid in the more general setting where $X$ is a smooth orbifold (or Deligne-Mumford stack) rather than a smooth algebraic variety. The previous discussion goes through in this situation with minimal changes as follows:

- We take $H_{X}$ to be the even part $^{2}$ of the Chen-Ruan orbifold cohomology $H_{\mathrm{CR}}^{\bullet}(X ; \mathbb{Q})$ rather than the even part of the ordinary cohomology $H^{\bullet}(X ; \mathbb{Q})$.

- We replace:

- the usual grading on $H^{\bullet}(X)$ by the age-shifted grading on $H_{\mathrm{CR}}^{\bullet}(X)$,

- the Poincaré pairing on $H^{\bullet}(X)$ by the orbifold Poincaré pairing on $H_{\mathrm{CR}}^{\bullet}(X)$. Note that $H^{2}(X) \subset H_{\mathrm{CR}}^{2}(X)$, and so definition (2) makes sense in the orbifold context.

- We define correlators (1) and (8) using orbifold Gromov-Witten invariants [1] rather than usual Gromov-Witten invariants. There are two small differences:

- a subtlety in the definition of $\mathrm{ev}_{k}^{\star}$, discussed in [1], [8, Section 2.2.2],

- the degree $d$ of an orbifold stable map $f: \Sigma \rightarrow X$ lies in $H_{2}(|X| ; \mathbb{Z})$, where $|X|$ is the coarse moduli space of $X$.

Having made these changes, the discussion in Sections 2.1-2.6 applies to orbifolds as well. In this context, the family of algebras $\left(H_{X} \otimes \Lambda, *\right)$ is called a quantum orbifold cohomology.

\footnotetext{
${ }^{2}$ Here we mean the even part of the rational cohomology of the inertia stack $I X$ with respect to the usual grading on $H^{\bullet}(I X)$, not the age-shifted grading.
} 


\subsection{FJRW Theory}

The discussion in this paper applies also to the so-called FJRW theory, which has been developed recently by Fan, Jarvis, and Ruan [15] based on an old idea of Witten [37]. FJRW theory is a Gromov-Witten-type theory with target a Landau-Ginzburg orbifold: it defines a cohomological field theory (CohFT) on a certain state space $H_{\text {FJRW }}$ that satisfies Teleman's homogeneity and flat vacuum conditions. Thus, Teleman's classification result applies to FJRW theory. FJRW theory differs from Gromov-Witten theory in that it lacks Novikov variables $Q_{1}, \ldots, Q_{r}$; most of the discussion in this paper, however, goes through just by setting $r=0$ :

- The genus-zero part of FJRW theory defines a Frobenius manifold structure on the formal neighborhood of the origin of $H_{\mathrm{FJRW}}$;

- Formal Semisimplicity (24), Genus-Zero Convegence (25), and Analytic Semisimplicity (26) make sense for this Frobenius manifold;

- The descendant potential $\mathcal{Z}_{\mathrm{FJRW}}$ is a formal power series in $\mathbf{t}(z) \in H_{\mathrm{FJRW}} \llbracket z \rrbracket$;

- The ancestor potential $\mathcal{A}_{\mathrm{FJRW}, t}$ is a formal power series in $\mathbf{y}(z) \in H_{\mathrm{FJRW}} \llbracket z \rrbracket$ and $t \in H_{\mathrm{FJRW}}$.

\section{Givental's Quantization Formalism}

In this section, we work over an arbitrary commutative ring $R$ that contains $\mathbb{Q}$. Let $V$ be a finitely generated free $R$-module equipped with a symmetric perfect pairing

$$
\langle\cdot, \cdot\rangle_{V}: V \otimes_{R} V \rightarrow R
$$

Let $\left\{\phi_{\alpha}\right\}_{\alpha=0}^{N}$ be an $R$-basis of $V$, and let $\phi^{\alpha}$ be the dual basis with respect to the pairing $\langle\cdot, \cdot\rangle_{V}$, so that $\left\langle\phi_{\alpha}, \phi^{\beta}\right\rangle_{V}=\delta_{\alpha}^{\beta}$. We denote a general point of $V \llbracket z \rrbracket$ by

$$
\mathbf{q}(z)=q_{0}+q_{1} z+q_{2} z+q_{3} z^{3}+\cdots
$$

and write $q_{i}=q_{i}^{0} \phi_{0}+\cdots+q_{i}^{N} \phi_{N}$. Then $\left\{q_{i}^{\alpha}: 0 \leq i<\infty, 0 \leq \alpha \leq N\right\}$ gives a coordinate system on $V \llbracket z \rrbracket$.

Remark 3.1. In the case where $R=\mathbb{Q}, V=H_{X}$, and $\langle\cdot, \cdot\rangle_{V}$ is the Poincare pairing, we recover the situation described in Section 2.6.

\subsection{Ancestor Fock Space}

Definition 3.2 (Ancestor Fock Space; see Givental [19, Sect. 8]). Choose a base point $-\delta=-\sum_{\alpha=0}^{N} \delta^{\alpha} \phi_{\alpha} \in V$, and consider the coordinate system $\left\{y_{i}^{\alpha}: 0 \leq i<\right.$ $\infty, 0 \leq \alpha \leq N\}$ on $V \llbracket z \rrbracket$ defined by

$$
y_{i}^{\alpha}= \begin{cases}q_{1}^{\alpha}+\delta^{\alpha} & \text { if } i=1, \\ q_{i}^{\alpha} & \text { otherwise. }\end{cases}
$$

Let $R \llbracket \mathbf{y} \rrbracket$ denote the formal power series ring $R \llbracket y_{i}^{\alpha}: 0 \leq i<\infty, 0 \leq \alpha \leq N \rrbracket$ equipped with the valuation $v$ defined by $v\left(y_{i}^{\alpha}\right)=i+1$. The ancestor Fock space 
$\mathfrak{F o c k}(V, \delta)$ is the set of elements

$$
\mathcal{A} \in R \llbracket \mathbf{y} \rrbracket\left\{\hbar^{-1}, \hbar \rrbracket\right.
$$

that admit an expansion of the form

$$
\mathcal{A}=\exp \left(\sum_{g=0}^{\infty} \hbar^{g-1} \mathcal{F}^{g}\right)
$$

such that $\mathcal{F}^{g} \in R \llbracket \mathbf{y} \rrbracket$ and

$$
\begin{aligned}
&\left.\mathcal{F}^{0}\right|_{\mathbf{y}(z)=0}=\left.\frac{\partial \mathcal{F}^{0}}{\partial y_{i}^{\alpha}}\right|_{\mathbf{y}(z)=0}=\left.\frac{\partial^{2} \mathcal{F}^{0}}{\partial y_{i_{1}}^{\alpha_{1}} \partial y_{i_{2}}^{\alpha_{2}}}\right|_{\mathbf{y}(z)=0}=0, \\
&\left.\mathcal{F}^{1}\right|_{\mathbf{y}(z)=0}=0,=0 \quad \text { whenever } i_{1}+\cdots+i_{n}>3 g-3+n . \\
&\left.\frac{\partial^{n} \mathcal{F}^{g}}{\partial y_{i_{1}}^{\alpha_{1}} \cdots \partial y_{i_{n}}^{\alpha_{n}}}\right|_{\mathbf{y}(z)=0}=0
\end{aligned}
$$

Write $y_{i}=y_{i}^{0} \phi_{0}+\cdots+y_{i}^{N} \phi_{N}$ and $\mathbf{y}(z)=\sum_{i=0}^{\infty} y_{i} z^{i}$. The coordinate system $\mathbf{y}=\left(y_{0}, y_{1}, y_{2}, \ldots\right)$ from Definition 3.2 is related to the coordinate system $\mathbf{q}=$ $\left(q_{0}, q_{1}, q_{2}, \ldots\right)$ defined before Remark 3.1 by

$$
q_{i}^{\alpha}= \begin{cases}y_{1}^{\alpha}-\delta^{\alpha} & \text { if } i=1 \\ y_{i}^{\alpha} & \text { otherwise }\end{cases}
$$

or in other words by $\mathbf{q}(z)=\mathbf{y}(z)-\delta z$; cf. the dilaton shift (11). Elements of $\mathfrak{F} \operatorname{ock}(V, \delta)$ can thus be regarded as functions on a formal neighborhood of the point $-\delta z \in V \llbracket z \rrbracket$.

REMARK 3.3. Any expression of the form (12) such that $\mathcal{F}^{g} \in R \llbracket \mathbf{y} \rrbracket$ and condition (13) holds is automatically an element of $R \llbracket \mathbf{y} \rrbracket\left\{\hbar^{-1}, \hbar \rrbracket\right.$.

Remark 3.4. Condition (13) implies that any element $\mathcal{A}$ of $\mathfrak{F o c k}(V, \delta)$ is tame in the sense of Givental [20]. Note in particular that $\mathcal{F}^{g}$ is a formal power series in the variables $y_{0}^{0}, \ldots, y_{0}^{N}, y_{1}^{0}, \ldots, y_{1}^{N}$ with coefficients in the polynomial ring $R\left[y_{i}^{\alpha}: 2 \leq i<\infty, 0 \leq \alpha \leq N\right]$.

Definition 3.5 (Rationality). An element $\mathcal{A}$ of $\mathfrak{F o c k}(V, \delta)$ is called rational if there exists a polynomial $P\left(q_{1}\right) \in R\left[V^{\vee}\right]$ with $P(-\delta)=1$ such that the potentials $\mathcal{F}^{g}$ from (12) satisfy

$$
\left.\frac{\partial^{n} \mathcal{F}^{g}}{\partial y_{i_{1}}^{\alpha_{1}} \cdots \partial y_{i_{n}}^{\alpha_{n}}}\right|_{\mathbf{y}(z)=y_{1} z}=f_{g, I, A}\left(q_{1}\right) P\left(q_{1}\right)^{-\left(5 g-5+2 n-\left(i_{1}+\cdots+i_{n}\right)\right)}
$$

for some polynomials $f_{g, I, A}\left(q_{1}\right) \in R\left[V^{\vee}\right]$ if $2 g-2+n>0$; here $I=\left(i_{1}, \ldots, i_{n}\right)$ and $A=\left(\alpha_{1}, \ldots, \alpha_{n}\right)$. We call $P$ a discriminant of $\mathcal{A}$. 
REMARK 3.6. Tameness (13) and rationality (15) for a potential can be summarized in the following expansion:

$$
\begin{aligned}
\mathcal{F}^{g}= & \delta_{g, 1} C^{(1)}\left(q_{1}\right) \\
& +\sum_{n: 2 g-2+n>0} \frac{1}{n !} \sum_{\substack{I: I=\left(i_{1}, \ldots, i_{n}\right) \\
i_{j} \neq 1 \text { for all } j \\
i_{1}+\cdots+i_{n} \leq 3 g-3+n}} \sum_{A=\left(\alpha_{1}, \ldots, \alpha_{n}\right)} C_{I, A}^{(g)}\left(q_{1}\right) q_{i_{1}}^{\alpha_{1}} \cdots q_{i_{n}}^{\alpha_{n}}
\end{aligned}
$$

with

$$
\begin{aligned}
C_{I, A}^{(g)}\left(q_{1}\right) & =f_{g, I, A}\left(q_{1}\right) P\left(q_{1}\right)^{-\left(5 g-5+2 n-\left(i_{1}+\cdots+i_{n}\right)\right)} \quad \text { and } \\
\frac{\partial C^{(1)}\left(q_{1}\right)}{\partial q_{1}^{\alpha}} & =f_{1,1, \alpha}\left(q_{1}\right) P\left(q_{1}\right)^{-1}
\end{aligned}
$$

for some polynomials $f_{g, I, A}\left(q_{1}\right) \in R\left[V^{\vee}\right]$. Note that $5 g-5+2 n-\left(i_{1}+\cdots+\right.$ $\left.i_{n}\right)=3 g-3+n-\left(i_{1}+\cdots+i_{n}\right)+2 g-2+n$ is always positive unless $(g, n)=$ $(1,0)$. The genus-one term $C^{(1)}\left(q_{1}\right)$ is in general not a rational function. See Remark 3.8 in the case of Gromov-Witten theory.

EXAmple 3.7. The total ancestor potential $\mathcal{A}_{X}$ of $X$ defines an element of the Fock space $\mathfrak{F o c k}\left(H_{X} \otimes \Lambda \llbracket t \rrbracket, \phi_{0}\right)$. Here the ground ring $R$ is $\Lambda \llbracket t \rrbracket$, the $R$-module $V$ is $H_{X} \otimes \Lambda \llbracket t \rrbracket$, and the pairing $\langle\cdot, \cdot\rangle_{V}$ is the Poincaré pairing, extended by $R$ linearity to take values in $R$. The dilaton shift discussed in Section 2.6 coincides with the identification (14). Tameness (13) follows from the dimension formula $\operatorname{dim} \overline{\mathcal{M}}_{g, m}=3 g-3+m$.

REMARK 3.8. The genus-one ancestor potential of a smooth projective variety $X$ satisfies [11]

$$
\left.\overline{\mathcal{F}}_{t}^{1}\right|_{\mathbf{y}(z)=y_{1} z}=-\frac{1}{24} \log \operatorname{sdet}\left(-q_{1} *_{t}\right),
$$

where $\operatorname{sdet}\left(-q_{1} *_{t}\right)$ denotes the superdeterminant of the quantum product on the total cohomology group $H^{\bullet}(X)=H^{\text {even }}(X) \oplus H^{\text {odd }}(X)$ (including the odd part). This follows from the localization of the integral to the locus of cycles of rational curves and $\int \overline{\mathcal{M}}_{1,1} \psi=1 / 24$. Therefore, the genus-one potential itself is not rational in $q_{1}$, but its derivatives are rational.

EXAMPLE 3.9. The ancestor potential $\mathcal{A}_{\mathrm{pt}}=\mathcal{A}_{t}$ of a point does not depend on $t \in H_{\mathrm{pt}}$ and coincides with the descendant potential $\mathcal{Z}_{\mathrm{pt}}$. This is called the WittenKontsevich tau-function and denoted by $\tau(\mathbf{q})$. It defines a rational element of the Fock space with $V=R=\mathbb{C}$ and $\delta=1$. In fact, applying the dilaton equation, we find that

$$
\begin{aligned}
& \left.\frac{\partial^{n} \mathcal{F}_{\mathrm{pt}}^{g}}{\partial y_{i_{1}} \cdots \partial y_{i_{n}}}\right|_{\mathbf{y}(z)=y_{1} z} \\
& \quad= \begin{cases}-\frac{1}{24} \log \left(-q_{1}\right) & \text { if } g=1 \text { and } n=0, \\
\left(-q_{1}\right)^{-(2 g-2+n)}\left\langle\psi_{1}^{i_{1}}, \ldots, \psi_{n}^{i_{n}}\right\rangle_{g, n, 0}^{\mathrm{pt}} & \text { otherwise. }\end{cases}
\end{aligned}
$$


Hence, we can take $P\left(q_{1}\right)=-q_{1}$. Note that $i_{1}+\cdots+i_{n}=3 g-3+n$ implies $2 g-2+n \leq 5 g-5+2 n-\left(i_{1}+\cdots+i_{n}\right)$.

REMARK 3.10. In view of Givental's formula (see Sections 4-5), we may speculate that in general the total ancestor potential of $X$ is rational with discriminant $\operatorname{det}\left(-q_{1} *\right)$ (determinant on the even part $H_{X}$ with even parameter $t \in H_{X}$ and even $q_{1} \in H_{X}$ ). We will prove that this is the case whenever the quantum cohomology of $X$ is semisimple; see Theorem 6.4.

REMARK 3.11. Givental's Lagrangian cone $\mathcal{L}_{X}$ (see [21]) has a singularity along a "divisor" that contains the vertex of the cone. Thus, it is natural to conjecture that the higher-genus descendant potentials of $X$ has poles only along that divisor. This is the rationality condition.

REMARK 3.12. Recall the definition of the genus- $g$ ancestor potential $\overline{\mathcal{F}}_{X}^{g}$ in (9). Consider the completion $\Upsilon$ of the polynomial ring $\mathbb{Q}\left[t^{0}, Q_{1} e^{t^{1}}, \ldots, Q_{r} e^{t^{r}}, t^{r+1}\right.$, $t^{r+2}, \ldots, t^{N}$ ] with respect to the valuation $v$ defined by

$$
\begin{aligned}
v\left(t_{i}\right)=1, & i=0 \text { or } r<i \leq N, \\
v\left(Q_{i} e^{t^{i}}\right)=1, & 1 \leq i \leq r .
\end{aligned}
$$

The divisor equation implies that $\overline{\mathcal{F}}_{X}^{g}$, which a priori is a formal power series in the variables $y_{j}^{\beta}$ with coefficients in

$$
\mathbb{Q} \llbracket Q_{1}, \ldots, Q_{r} \rrbracket \llbracket t^{0}, \ldots, t^{N} \rrbracket
$$

is in fact a formal power series in the variables $y_{j}^{\beta}$ with coefficients in $\Upsilon$. Thus, the total ancestor potential $\mathcal{A}_{X}$ defines an element of the Fock space $\mathfrak{F o c k}\left(H_{X} \otimes\right.$ $\left.\Upsilon, \phi_{0}\right)$.

Definition 3.13. For $\epsilon>0$, define $\Upsilon_{\epsilon}$ to be the subring of $\Upsilon$ consisting of elements in $\Upsilon$ that converge on the region

$$
\left\{\left|t^{0}\right|<\epsilon,\left|Q_{1} e^{t^{1}}\right|<\epsilon, \ldots,\left|Q_{r} e^{t^{r}}\right|<\epsilon,\left|t^{r+1}\right|<\epsilon, \ldots,\left|t^{N}\right|<\epsilon\right\} .
$$

The ancestor Gromov-Witten potential $\mathcal{A}_{X}$ is said to be convergent if it is a rational element of $\mathfrak{F o c k}\left(H_{X} \otimes \Upsilon_{\epsilon}, \phi_{0}\right)$ for some $\epsilon>0$.

Remark 3.14. When $\mathcal{A}_{X}$ is convergent in the sense of Definition 3.13, each genus- $g$ ancestor potential $\overline{\mathcal{F}}_{X}^{g}$ (see equation (9)) is a power series in the variables $y_{j}^{\beta}$ with coefficients in $\Upsilon_{\epsilon}$. Furthermore, in this case, $\left.\overline{\mathcal{F}}_{X}^{g}\right|_{Q_{1}=\cdots=Q_{r}=1}$ is a formal power series in $y_{j}^{\beta}$ with coefficients in analytic functions on $\mathcal{M}$, where $\mathcal{M}$ is a neighborhood (4) of the large-radius limit point.

\subsection{Propagator}

Let $\left(V,\langle\cdot, \cdot\rangle_{V}\right)$ and $\left(W,\langle\cdot, \cdot\rangle_{W}\right)$ be free $R$-modules with symmetric perfect pairings. 
Definition 3.15. The Givental symplectic form $\Omega_{V}$ is the antisymmetric bilinear form on $V((z))$ defined by

$$
\Omega_{V}\left(f_{1}, f_{2}\right)=\operatorname{Res}_{z=0}\left\langle f_{1}(-z), f_{2}(z)\right\rangle_{V} d z .
$$

Notation 3.16. An $R \llbracket z \rrbracket-$ linear isomorphism $A: V \llbracket z \rrbracket \rightarrow W \llbracket z \rrbracket$ can be expressed uniquely in the form $A=A_{0}+A_{1} z+A_{2} z^{2}+\cdots$ where $A_{k} \in \operatorname{Hom}_{R}(V$, $W)$. We write the coefficients of this expansion as $A_{k}, k \geq 0$, and write $A$ as $A(z)$ when we wish to emphasize the dependence on $z$.

Definition 3.17. An isomorphism $A: V \llbracket z \rrbracket \rightarrow W \llbracket z \rrbracket$ is said to be unitary if it is $R \llbracket z \rrbracket-$ linear and satisfies

$$
\left\langle A(-z) v_{1}, A(z) v_{2}\right\rangle_{W}=\left\langle v_{1}, v_{2}\right\rangle_{V}
$$

for all $v_{1}, v_{2} \in V$.

REMARK 3.18. An $R \llbracket z \rrbracket$-linear isomorphism $A: V \llbracket z \rrbracket \rightarrow W \llbracket z \rrbracket$ is unitary if and only if the map $V((z)) \rightarrow W((z))$ induced by $A$ intertwines the Givental symplectic forms.

Definition 3.19 (Propagator; cf. Givental [19]). Let $A: V \llbracket z \rrbracket \rightarrow W \llbracket z \rrbracket$ be a unitary isomorphism. The propagator for $A$ is a bivector field $\Delta$ on $V \llbracket z \rrbracket$ defined by

where

$$
\Delta=\sum_{i, j=0}^{\infty} \sum_{\alpha, \beta=0}^{N} \Delta^{(i, \alpha),(j, \beta)} \frac{\partial}{\partial q_{i}^{\alpha}} \frac{\partial}{\partial q_{j}^{\beta}},
$$

$$
\sum_{i, j=0}^{\infty} \Delta^{(i, \alpha),(j, \beta)}(-1)^{i+j} w^{i} z^{j}=\left\langle\phi^{\alpha}, \frac{A(w)^{\dagger} A(z)-\mathrm{Id}}{z+w} \phi^{\beta}\right\rangle_{V} .
$$

Here the coordinates $q_{i}^{\alpha}$ and the basis $\left\{\phi^{\alpha}\right\}$ are defined before Remark 3.1; $\Delta$ is in fact independent of choice of basis. Also, $A(z)^{\dagger}: W \llbracket z \rrbracket \rightarrow V \llbracket z \rrbracket$ denotes the adjoint of $A(z)$ with respect to the pairings $\langle\cdot, \cdot\rangle_{V}$ and $\langle\cdot, \cdot\rangle_{W}$ (since $A(z)$ is unitary, we have $\left.A(z)^{\dagger}=A(-z)^{-1}\right)$.

\subsection{Quantized Operator}

Let $A: V \llbracket z \rrbracket \rightarrow W \llbracket z \rrbracket$ be a unitary isomorphism. Recall the definition of $A_{0}$ in Notation 3.16. We define the quantized operator

$$
\widehat{A}: \mathfrak{F o c k}(V, \delta) \rightarrow \mathfrak{F o c k}\left(W, A_{0}(\delta)\right)
$$

as follows. For a given element $\mathcal{A} \in \mathfrak{F} \mathfrak{o c k}(V, \delta)$, we set

$$
\widetilde{\mathcal{A}}=\exp \left(\frac{\hbar}{2} \Delta\right) \mathcal{A} \in \mathfrak{F o c k}(V, \delta)
$$

and then push $\widetilde{\mathcal{A}}$ forward along the identification $A(z): V \llbracket z \rrbracket \cong W \llbracket z \rrbracket$ :

$$
(\widehat{A} \mathcal{A})(\mathbf{q}):=\widetilde{\mathcal{A}}\left(A(z)^{-1} \mathbf{q}(z)\right) .
$$


Proposition 3.20. The quantized operator $\widehat{A}$ is well defined. Moreover, if $\mathcal{A}$ is a rational element of $\mathfrak{F o c k}(V, \delta)$ with discriminant $P\left(q_{1}\right) \in R\left[V^{\vee}\right]$, then $\widehat{A} \mathcal{A}$ is a rational element of $\mathfrak{F o c k}\left(W, A_{0}(\delta)\right)$ with discriminant $P\left(A_{0}^{-1} q_{1}\right) \in R\left[W^{\vee}\right]$.

Proof. The first claim was proved by Givental using a Feynman diagram argument [20, Prop. 5]. It remains to show that the quantized operator $\widehat{A}$ preserves rationality and to calculate its effect on the discriminant. Recall that $\widetilde{\mathcal{A}}=\exp \left(\frac{\hbar}{2} \Delta\right) \mathcal{A}$, and define $\widetilde{\mathcal{F}}^{g}$ by

$$
\widetilde{\mathcal{A}}=\exp \left(\sum_{g=0}^{\infty} \hbar^{g-1} \tilde{\mathcal{F}}^{g}\right) .
$$

Following Givental's proof, we express

$$
\left.\frac{\partial^{n} \widetilde{\mathcal{F}}^{g}}{\partial y_{i_{1}}^{\alpha_{1}} \cdots \partial y_{i_{n}}^{\alpha_{n}}}\right|_{\mathbf{y}(z)=y_{1} z}
$$

as a sum over decorated Feynman graphs. These decorated Feynman graphs are connected multigraphs, in which loops are allowed, such that

- each vertex $v$ is labeled by an integer $g_{v} \geq 0$;

- a label $(j, \beta) \in \mathbb{Z}_{\geq 0} \times\{0, \ldots, N\}$ is assigned to each pair of a vertex and an edge incident to it (for an edge-loop, we distinguish the two ends of the edge);

- the graph has $n$ external edges, called legs, labeled by $\left(i_{1}, \alpha_{1}\right), \ldots,\left(i_{n}, \alpha_{n}\right)$;

- the Euler number $\chi$ of the graph satisfies $g=1-\chi+\sum_{v \text { :vertex }} g_{v}$;

and such that the following stability condition holds: for each vertex $v$, if $\left(j_{1}, \beta_{1}\right), \ldots,\left(j_{m}, \beta_{m}\right)$ are all the labels attached to the edges or legs incident to $v$, then

$$
j_{1}+\cdots+j_{m} \leq 3 g_{v}-3+m .
$$

Givental's original argument shows that the number of such decorated Feynman graphs is finite. Let $\Gamma$ be a decorated Feynman graph as above, and let $V(\Gamma), E(\Gamma)$ be respectively the set of vertices and the set of edges of $\Gamma$. The contribution of $\Gamma$ to $(19)$ is

$$
\frac{1}{|\operatorname{Aut}(\Gamma)|} \prod_{e \in E(\Gamma)}(\text { edge term for } e) \prod_{v \in V(\Gamma)}(\text { vertex term for } v),
$$

where the edge term for an edge with labels $(i, \alpha),(j, \beta)$ is $\Delta^{(i, \alpha),(j, \beta)}$, and the vertex term for a vertex $v$ with labels $\left(j_{1}, \beta_{1}\right), \ldots,\left(j_{m}, \beta_{m}\right)$ is

$$
\left.\frac{\partial^{n} \mathcal{F}^{g_{v}}}{\partial y_{j_{1}}^{\beta_{1}} \cdots \partial y_{j_{m}}^{\beta_{m}}}\right|_{\mathbf{y}(z)=y_{1} z} .
$$

We write $n_{v}=m$ and $d_{v}=j_{1}+\cdots+j_{m}$. Suppose that $\mathcal{A}$ is rational with discriminant $P\left(q_{1}\right)$. The partial derivative (19) is a finite sum of terms (20), and each vertex term (21) takes the form

$$
\frac{f_{v}\left(q_{1}\right)}{P\left(q_{1}\right)^{5 g_{v}-5+2 n_{v}-d_{v}}},
$$


where $f_{v}$ is a polynomial. Using the (in)equalities

$$
\begin{aligned}
\sum_{v \in V(\Gamma)}\left(g_{v}-1\right) & =g-1-|E(\Gamma)|, \quad \sum_{v \in V(\Gamma)} n_{v}=2|E(\Gamma)|+n, \\
\sum_{v \in V(\Gamma)} d_{v} & \geq i_{1}+\cdots+i_{n}
\end{aligned}
$$

we have

$$
\sum_{v \in V(\Gamma)}\left(5 g_{v}-5+2 n_{v}-d_{v}\right) \leq 5 g-5+2 n-\left(i_{1}+\cdots+i_{n}\right)
$$

Hence, each term (20) is a rational function with denominator

$$
P\left(q_{1}\right)^{5 g-5+2 n-\left(i_{1}+\cdots+i_{n}\right)} \text {. }
$$

It follows that $\tilde{\mathcal{A}}$ is rational with discriminant $P\left(q_{1}\right) \in R\left[V^{\vee}\right]$. The change of variables $\mathbf{q}(z) \rightarrow A(z)^{-1} \mathbf{q}(z)$ preserves tameness and rationality: we can easily check that the expansion in Remark 3.6 is preserved. Thus, $\widehat{A} \mathcal{A}$ is rational, with discriminant $P\left(A_{0}^{-1} q_{1}\right) \in R\left[W^{\vee}\right]$.

EXAMPLE 3.21. Figure 1 shows an example of a decorated Feyman diagram $\Gamma$. This graph $\Gamma$ has one leg, labeled by $(p, \xi)$; it occurs in the Feynman sum for

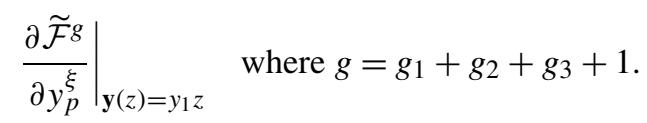

The stability condition asserts that $i \leq 3 g_{1}-2, j+k+p \leq 3 g_{2}$, and $l+m+n \leq$ $3 g_{3}$. The automorphism group of $\Gamma$ is trivial if $(m, \rho) \neq(n, \mu)$ and is equal to $\mathbb{Z} / 2 \mathbb{Z}$ if $(m, \rho)=(n, \mu)$. Thus, the contribution of $\Gamma$ to the Feynman sum is equal to

$$
\left.\Delta^{(i, \alpha),(j, \beta)} \Delta^{(k, \gamma),(l, \epsilon)} \Delta^{(m, \rho),(n, \mu)}\left(\frac{\partial \mathcal{F}^{g_{1}}}{\partial y_{i}^{\alpha}} \frac{\partial^{3} \mathcal{F}^{g_{2}}}{\partial y_{j}^{\beta} \partial y_{k}^{\gamma} \partial y_{p}^{\xi}} \frac{\partial^{3} \mathcal{F}^{g_{3}}}{\partial y_{l}^{\epsilon} \partial y_{m}^{\rho} \partial y_{n}^{\mu}}\right)\right|_{\mathbf{y}(z)=y_{1} z}
$$

if $(m, \rho) \neq(n, \mu)$ and is equal to half of this if $(m, \rho)=(n, \mu)$.

REMARK 3.22. Let $\left(U,\langle\cdot, \cdot\rangle_{U}\right)$ be another free $R$-module with a perfect pairing. Let $A: V \llbracket z \rrbracket \rightarrow W \llbracket z \rrbracket$ and $B: W \llbracket z \rrbracket \rightarrow U \llbracket z \rrbracket$ be unitary isomorphisms. Then we can define three propagators $\Delta^{A}, \Delta^{B}, \Delta^{B A}$ corresponding to the maps $A, B$, $B A$, respectively. The bivector fields $\Delta^{A}$ on $V \llbracket z \rrbracket, \Delta^{B}$ on $W \llbracket z \rrbracket$, and $\Delta^{B A}$ on $V \llbracket z \rrbracket$ satisfy

$$
\Delta^{B A}=\Delta^{A}+A(z)^{*} \Delta^{B},
$$

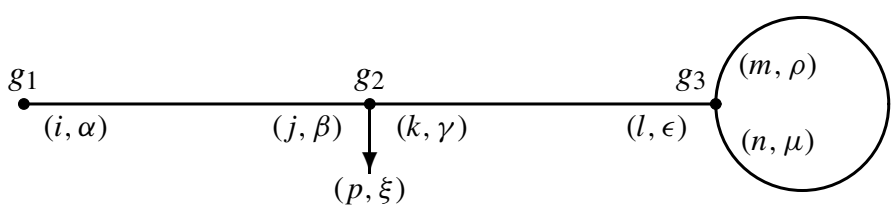

Figure 1 A decorated graph with one leg 
where $A(z)^{*} \Delta^{B}$ means the pull-back of the bivector field $\Delta^{B}$ on $W \llbracket z \rrbracket$ by the isomorphism $A(z): V \llbracket z \rrbracket \cong W \llbracket z \rrbracket$. Therefore,

$$
(B A)^{\wedge}=\widehat{B} \widehat{A}
$$

as a map from $\mathfrak{F o c k}(V, \delta)$ to $\mathfrak{F o c k}\left(U, B_{0} A_{0}(\delta)\right)$.

\section{Givental's Formula in the Analytic Setting}

Let $\mathcal{M}$ be an analytic Frobenius manifold over $\mathbb{C}$. This comprises the following data: a smooth complex analytic space $\mathcal{M}$; a flat metric ${ }^{3} g$ on $\mathcal{M}$; a product $*_{t}$ on each tangent space $T_{t} \mathcal{M}$, varying analytically with $t$; a flat identity vector field 1; a vector field $E$ on $\mathcal{M}$ called the Euler vector field; and an integer $D$ called the conformal dimension. These structures are required to satisfy a number of conditions: see [13, Def. 1.2]. In particular, $\left(T_{t} \mathcal{M}, *_{t}, g\right)$ forms a family of commutative associative Frobenius algebras, varying analytically with $t$, and $\nabla^{\mathrm{LC}}\left(\nabla^{\mathrm{LC}} E\right)=0$ where $\nabla^{\mathrm{LC}}$ is the Levi-Civita connection defined by $g$. The operator $\mu: T \mathcal{M} \rightarrow T \mathcal{M}$ defined by $\mu=\left(1-\frac{D}{2}\right) \mathrm{Id}-\nabla^{\mathrm{LC}} E$ is called the grading operator. One example of an analytic Frobenius manifold over $\mathbb{C}$ is given by the quantum cohomology of a smooth variety $X$ such that the genus-zero GromovWitten potential converges in the sense of Section 2.3; in this case, $\mathcal{M}$ is the neighborhood (4) of the large-radius limit point.

Suppose further that $\mathcal{M}$ is generically semisimple, that is, that $\left(T_{t} \mathcal{M}, *_{t}\right)$ is a semisimple algebra for generic $t \in \mathcal{M}$, and fix a semisimple point $t$. The eigenvalues of multiplication $(E *)$ by the Euler vector field form canonical coordinates $u^{0}, \ldots, u^{N}$ on a neighborhood of $t$. The vector fields $\partial / \partial u^{i} \in T \mathcal{M}$ are then the idempotents in the semisimple algebra $(T \mathcal{M}, *)$ in a neighborhood of $t$. Let

$$
\Delta^{i}(t)=\left(g\left(\left.\frac{\partial}{\partial u^{i}}\right|_{t},\left.\frac{\partial}{\partial u^{i}}\right|_{t}\right)\right)^{-1} .
$$

Proposition 4.1 (Dubrovin [14, Lecture 4], Teleman [36, Thm. 8.15]). At the semisimple point $t \in \mathcal{M}$, the equation

$$
\left(z \frac{\partial}{\partial z}+\frac{1}{z}\left(E *_{t}\right)+\mu\right) S=0
$$

has a unique solution of the form $S=\Psi_{t} R_{t} \exp (U / z)$ such that:

(1) $\Psi_{t} \in \operatorname{Hom}\left(\mathbb{C}^{N+1}, T_{t} \mathcal{M}\right)$ is the isomorphism $\mathbb{C}^{N+1} \cong T_{t} \mathcal{M}$ that sends the ith standard basis vector in $\mathbb{C}^{N+1}$ to the ith normalized idempotent $\sqrt{\Delta^{i}(t)} \partial / \partial u^{i} \in T_{t} \mathcal{M}$

(2) $R_{t} \in \operatorname{End}\left(\mathbb{C}^{N+1}\right) \otimes \mathbb{C} \llbracket z \rrbracket$ with $R_{t} \equiv \mathrm{Id} \bmod z$;

(3) $U=\operatorname{diag}\left(u^{0}, \ldots, u^{N}\right)$ where $u^{0}, \ldots, u^{N}$ are the eigenvalues of $E *_{t}$.

The transformation $R_{t}$ satisfies

$$
R_{t}(-z)^{\mathrm{T}} R_{t}(z)=\mathrm{Id},
$$

\footnotetext{
${ }^{3}$ Metric here means $\mathbb{C}$-bilinear quadratic form on each tangent space $T_{t} M$, varying analytically with $t$.
} 
where $R_{t}(-z)^{\mathrm{T}}$ means the transpose of $R_{t}(-z)$ (regarded as an $(N+1) \times(N+1)$ matrix).

The transformations $\Psi$ and $R$ in Proposition 4.1 coincide with those defined by Givental [18, Sect. 1.3], although his definitions are different as he is working in a setting where there may be no Euler vector field. As Dubrovin observed, $\Psi_{t} R_{t} \exp (U / z)$ is automatically flat with respect to the Dubrovin connection as $t$ varies, and, as $t$ varies, $R_{t}$ is automatically homogeneous with respect to the Euler vector field $E=\sum_{i=0}^{N} u^{i} \partial / \partial u^{i}$ :

$$
\left(z \frac{\partial}{\partial z}+\sum_{i} u^{i} \frac{\partial}{\partial u^{i}}\right) R_{t}=0 .
$$

We regard the composite map $\Psi_{t} R_{t}$ as giving a unitary isomorphism $\mathbb{C}^{N+1} \llbracket z \rrbracket \rightarrow T_{t} \mathcal{M} \llbracket z \rrbracket$ where $\mathbb{C}^{N+1}$ is endowed with the standard inner product (see Definition 3.17). In view of Example 3.9, we know that the product of Witten-Kontsevich $\tau$-functions

$$
\mathcal{T}=\prod_{\alpha=0}^{N} \tau\left(\mathbf{q}^{\alpha}\right) \quad \text { where }\left(\mathbf{q}^{0}, \ldots, \mathbf{q}^{N}\right) \in \mathbb{C}^{N+1} \llbracket z \rrbracket
$$

lies in the Fock space $\mathfrak{F o c k}\left(\mathbb{C}^{N+1},(1, \ldots, 1)\right)$. It is rational with the discriminant $P\left(q_{1}^{0}, \ldots, q_{1}^{N}\right)=\prod_{\alpha=0}^{N}\left(-q_{1}^{\alpha}\right)$.

Definition 4.2 (Givental [19, Sect. 6.8]). The abstract ancestor potential $\mathcal{A}_{t}^{\mathrm{abs}}$ is

$$
\mathcal{A}_{t}^{\mathrm{abs}}=e^{-(1 / 48) \sum_{i} \log \Delta^{i}(t)} \widehat{\Psi_{t}} \widehat{R}_{t}(\mathcal{T}) .
$$

When the semisimple point $t \in \mathcal{M}$ is clear from context, we will write $\mathcal{A}^{\text {abs }}$ instead of $\mathcal{A}_{t}^{\mathrm{abs}}$.

Proposition 4.3. The abstract ancestor potential $\mathcal{A}_{t}^{\mathrm{abs}}$ is a well-defined rational element of $\mathfrak{F o c k}\left(T_{t} \mathcal{M}, \mathbf{1}\right)$ with discriminant $\operatorname{det}\left(-q_{1} *_{t}\right)$.

Proof. We first observe that the right-hand side of (23) is unambiguous. The matrices $\Psi_{t}$ and $R_{t}$ depend on

- a choice of ordering of the canonical co-ordinates $u^{0}, \ldots, u^{N}$ at $t$, and

- the choice of square roots $\sqrt{\Delta^{i}(t)}$.

Thus, any two different choices of $\Psi_{t} R_{t}$ are related by right multiplication by a signed permutation matrix. Now $\mathcal{T}$ is almost invariant under a signed permutation $\left(\mathbf{q}^{0}, \ldots, \mathbf{q}^{N}\right) \mapsto\left( \pm \mathbf{q}^{\sigma(0)}, \ldots, \pm \mathbf{q}^{\sigma(N)}\right)$ : the only noninvariant part is the genus-one $\log$-term $-\frac{1}{24} \sum_{\alpha} \log \left(-q_{1}^{\alpha}\right)$. The constant ambiguity in this genus-one term cancels with the ambiguity of $-\frac{1}{48} \sum_{i} \log \Delta^{i}(t)$; the genus-one term $\mathcal{F}_{\text {abs }}^{1}$ in $\log \mathcal{A}_{t}^{\mathrm{abs}}$ is normalized by the condition

$$
\mathcal{F}_{\text {abs }}^{1} \mid \mathbf{y}(z)=0=0 .
$$

Thus, $\mathcal{A}_{t}^{\mathrm{abs}}$ is independent of all choices. 
Proposition 3.20 implies that $\widehat{\Psi_{t}} \widehat{R}_{t}(\mathcal{T})$ is a rational element of $\mathfrak{F o c k}\left(T_{t} \mathcal{M}\right.$, $\left.\left.\sum_{i=0}^{N} \sqrt{\Delta^{i}(t)} \partial / \partial u^{i}\right)\right)$ with discriminant

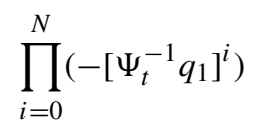

where $q_{1} \in T_{t} \mathcal{M}$. Because $\widehat{\Psi}_{t} \widehat{R}_{t}(\mathcal{T})$ is rational, $\mathcal{A}_{t}^{\text {abs }}=e^{-(1 / 48) \sum_{i} \log \Delta^{i}(t)} \times$ $\widehat{\Psi}_{t} \widehat{R}_{t}(\mathcal{T})$ can naturally be regarded, via analytic continuation, as an element of $\mathfrak{F o c k}\left(T_{t} \mathcal{M}, \mathbf{1}\right)$. We can normalize the discriminant by the nonzero factor $e^{(1 / 2) \sum_{i} \log \Delta^{i}(t)}$ :

$$
\begin{aligned}
P\left(q_{1}\right) & =e^{(1 / 2) \sum_{i} \log \Delta^{i}(t)} \prod_{i=0}^{N}\left(-\left[\Psi_{t}^{-1} q_{1}\right]^{i}\right) \\
& =\operatorname{det}\left(-q_{1} *_{t}\right),
\end{aligned}
$$

so that $P(-\mathbf{1})=1$.

Remark 4.4. When $t$ varies, $\mathcal{A}_{t}^{\text {abs }}$ defines a rational element of $\mathfrak{F o c k}(T \mathcal{M}(U), \mathbf{1})$ with $U$ a neighborhood of $t$. Here $T \mathcal{M}(U)$ is regarded as a free $\mathcal{O}(U)$-module.

Remark 4.5. The transformation $R_{t}=I+R_{1}(t) z+R_{2}(t) z^{2}+\cdots$ in Proposition 4.1 can be determined by solving the equations

$$
\left(z \frac{\partial}{\partial z}+\frac{1}{z}\left(E *_{t}\right)+\mu\right) \Psi_{t} R_{t} \exp \left(\frac{U}{z}\right)=0
$$

order by order in $z$. It follows, and this will be important below, that if the canonical co-ordinates $u^{i}$ and the matrix entries of $\Psi_{t}$ all lie in some field of functions $k$, then the entries of each matrix $R_{i}(t)$ lie in $k$ too.

\section{Givental's Formula in the Formal Setting}

Note that the discussion in Section 4 makes sense, and the analog of Proposition 4.1 holds, in the setting where $\mathcal{M}$ is a formal Frobenius manifold over an algebraically closed field $k$ of characteristic zero. In this case, $\mathcal{M}$ is the formal neighborhood of zero in a vector space $H$, so $\mathcal{M}=\operatorname{Spf} k \llbracket s^{0}, \ldots, s^{N} \rrbracket$ where $\phi_{0}, \ldots, \phi_{N}$ is a basis for $H$ and $s=s^{0} \phi_{0}+\cdots+s^{N} \phi_{N}$ is a point of $H$. The family of products on the tangent spaces to $\mathcal{M}$ give (and are given by) a $k \llbracket s^{0}, \ldots, s^{N} \rrbracket-$ bilinear product $*$ on $H \llbracket s^{0}, \ldots, s^{N} \rrbracket$. We choose $\phi_{0}$ to be the identity of the product $*$. A formal Frobenius manifold is said to be semisimple at the origin if the algebra $\left(H,\left.*\right|_{s=0}\right)$ is semisimple. (The origin is in any case the only $k$ valued point of $\mathcal{M}$.) Then, since $k$ is algebraically closed, distinct eigenvalues $u^{0}, \ldots, u^{N}$ for $(E *)$ exist in $k \llbracket s^{0}, \ldots, s^{N} \rrbracket$; these form canonical coordinates on a formal neighborhood of $s=0$ in $\mathcal{M}$. The vectors $\partial / \partial u^{i}$ are idempotents in the algebra $\left(H \llbracket s^{0}, \ldots, s^{N} \rrbracket, *\right)$, and we define $\Delta^{i} \in k \llbracket s^{0}, \ldots, s^{N} \rrbracket$ by

$$
\Delta^{i}=\left(g\left(\frac{\partial}{\partial u^{i}}, \frac{\partial}{\partial u^{i}}\right)\right)^{-1} .
$$


For Proposition 4.1 we replace:

$$
\begin{aligned}
& \Psi_{u} \in \operatorname{Hom}\left(\mathbb{C}^{N+1}, T_{u} \mathcal{M}\right) \quad \text { by } \Psi \in \operatorname{Hom}\left(k^{N+1}, H\right) \llbracket s^{0}, \ldots, s^{N} \rrbracket, \\
& R_{u} \in \operatorname{End}\left(\mathbb{C}^{N+1}\right) \otimes \mathbb{C} \llbracket z \rrbracket \quad \text { by } R \in \operatorname{End}\left(k^{N+1}\right) \llbracket z \rrbracket \llbracket s^{0}, \ldots, s^{N} \rrbracket,
\end{aligned}
$$

with the rest of the conditions unchanged. In other words: the canonical coordinates $u^{i}$, the normalizations $\Delta^{i}$, and the transformations $\Psi$ and $R$ are all defined in a formal neighborhood of $s=0$ in $\mathcal{M}$.

Proposition 5.1 (Formal version of Proposition 4.1). The equation

$$
\left(z \frac{\partial}{\partial z}+\frac{1}{z}(E *)+\mu\right) S=0
$$

has a unique solution of the form $S=\Psi R \exp (U / z)$ such that:

(1) $\Psi \in \operatorname{Hom}\left(k^{N+1}, H\right) \llbracket s^{0}, \ldots, s^{N} \rrbracket$ sends the ith standard basis vector in $k^{N+1}$ to the ith normalized idempotent $\sqrt{\Delta^{i}} \partial / \partial u^{i} \in H \llbracket s^{0}, \ldots, s^{N} \rrbracket ;$

(2) $R \in \operatorname{End}\left(k^{N+1}\right) \llbracket z \rrbracket \llbracket s^{0}, \ldots, s^{N} \rrbracket$ with $R \equiv \operatorname{Id} \bmod z$;

(3) $U=\operatorname{diag}\left(u^{0}, \ldots, u^{N}\right)$.

The transformation $R$ satisfies ${ }^{4}$

$$
R(-z)^{\mathrm{T}} R(z)=\mathrm{Id} .
$$

The composition $\Psi R: k^{N+1} \llbracket s^{0}, \ldots, s^{N} \rrbracket \llbracket z \rrbracket \rightarrow H \llbracket s^{0}, \ldots, s^{N} \rrbracket \llbracket z \rrbracket$ is a unitary isomorphism (see Definition 3.17) over the ground ring $k \llbracket s^{0}, \ldots, s^{N} \rrbracket$; thus, the following definition makes sense.

Definition 5.2 (Formal version of Definition 4.2). The abstract ancestor potential $\mathcal{A}_{s}^{\mathrm{abs}}$ is

$$
\mathcal{A}_{s}^{\mathrm{abs}}=\left(\prod_{i=0}^{i=N} \Delta^{i}\right)^{-1 / 48} \widehat{\Psi} \widehat{R}(\mathcal{T}) .
$$

Just as in Proposition 4.3, $\mathcal{A}_{s}^{\text {abs }}$ is a well-defined rational element of $\mathfrak{F o c k}\left(H \llbracket s^{0}\right.$, $\left.\ldots, s^{N} \rrbracket, \phi_{0}\right)$ with discriminant $P\left(q_{1}\right)=\operatorname{det}\left(-q_{1} *\right) \in k \llbracket s^{0}, \ldots, s^{N} \rrbracket\left[q_{1}^{0}, \ldots\right.$, $\left.q_{1}^{N}\right]$.

\section{Teleman Implies Givental}

Let $X$ be a smooth projective toric variety. Recall the definition of the total ancestor potential $\mathcal{A}_{X}$ in equation (10). The genus-zero Gromov-Witten potential $F_{X}^{0}$ converges [25] in the sense of Section 2.3, and so the quantum cohomology of $X$ defines an analytic Frobenius manifold (see Sect. 4). This Frobenius manifold is semisimple [25]. When $X$ is a Fano toric variety, Givental proves that

$$
\left.\mathcal{A}_{X}\right|_{Q_{1}=\cdots=Q_{r}=1}=\mathcal{A}^{\mathrm{abs}}
$$

\footnotetext{
${ }^{4}$ As in the analytic case, the transformation $R$ here is in addition automatically flat with respect to the Dubrovin connection and homogeneous with respect to the Euler vector field.
} 
by establishing a similar formula in the equivariant Gromov-Witten theory of $X$ and then taking a nonequivariant limit. His argument simultaneously proves:

(A) The convergence of $\left.\mathcal{A}_{X}\right|_{Q_{1}=\ldots=Q_{r}=1}$ in the sense of Definition 3.13;

(B) The equality $\left.\mathcal{A}_{X}\right|_{Q_{1}=\cdots=Q_{r}=1}=\mathcal{A}^{\text {abs }}$, where the right-hand side is defined as in Section 4.

Givental conjectured that (A) and (B) hold in general. His calculation in equivariant Gromov-Witten theory in fact applies to any smooth projective toric variety $X$, and Iritani [25] has proven that we can take the nonequivariant limit of this calculation even if $X$ is not Fano, so that (A) and (B) are known to hold whenever $X$ is a smooth projective toric variety.

In this section we explain how Givental's statements (A) and (B) can be deduced in much greater generality from Teleman's classification of DeligneMumford field theories (DMTs) [36]. Teleman proves [36, Thm. 1] that if a DMT satisfies

- a Cohomological Field Theory condition,

- a homogeneity condition (involving an Euler vector field),

- a flat vacuum condition (involving the identity element of the Frobenius algebra),

and if its genus-zero part defines a semisimple Frobenius algebra, then:

- the DMT can be uniquely reconstructed from its genus-zero part, and

- the ancestor potential of the DMT coincides with Givental's abstract potential $\mathcal{A}^{\mathrm{abs}}$.

Teleman's argument works over an arbitrary field of characteristic zero.

We now consider three conditions on the Gromov-Witten invariants of a projective variety $X$. Let $k$ denote the algebraic closure of the fraction field of $\Lambda \llbracket t \rrbracket$. The first condition, which we call Formal Semisimplicity, is

the quantum cohomology algebra $\left(H_{X} \otimes k, *\right)$ is semisimple.

The second condition, which we call Genus-Zero Convergence, is

the genus-zero Gromov-Witten potential $F_{X}^{0}$ converges in the sense of Section 2.3.

Let $\mathcal{M} \subset H_{X} \otimes \mathbb{C}$ be a neighborhood (4) of the large-radius limit point. If GenusZero Convergence holds, then, as discussed in Section 4, the genus-zero GromovWitten theory of $X$ defines on $\mathcal{M}$ the structure of an analytic Frobenius manifold over $\mathbb{C}$. The third condition, which we call Analytic Semisimplicity, is

this analytic Frobenius manifold is generically semisimple.

Remark 6.1. Formal Semisimplicity (24) and Genus-Zero Convergence (25) together imply Analytic Semisimplicity (26), and Genus-Zero Convergence (25) and Analytic Semisimplicity (26) together imply Formal Semisimplicity (24).

REMARK 6.2. All three conditions are satisfied when $X$ is a smooth projective toric variety; this follows from mirror symmetry for toric varieties $[17 ; 24 ; 25]$. 
Remark 6.3. If both Genus-Zero Convergence (25) and Analytic Semisimplicity (26) hold, then we can define the abstract ancestor potential $\mathcal{A}_{\text {an }}^{\text {abs }}$ as in Section 4. The subscript "an" here is to emphasize that we are working in the analytic setting.

In Section 6.1 we will show that if Formal Semisimplicity holds, then we can apply Teleman's theorem to the Gromov-Witten theory of $X$, thereby recovering the total ancestor potential $\mathcal{A}_{X}$ from the quantum cohomology. In Section 6.2 we show that if both Genus-Zero Convergence and Analytic Semisimplicity hold, then the total ancestor potential $\mathcal{A}_{X}$ is convergent in the sense of Definition 3.13, and is equal to the abstract ancestor potential $\mathcal{A}_{\text {an }}^{\text {abs }}$.

\subsection{Applying Teleman's Theorem in the Formal Setting}

Recall that $k$ denotes the algebraic closure of the fraction field of $\Lambda \llbracket t \rrbracket$. The quantum cohomology $\left(H_{X} \otimes k, *\right)$ over $k$ is equipped with the element

$$
E=t^{0} \phi_{0}+c_{1}(X)+\sum_{i=r+1}^{N}\left(1-\frac{1}{2} \operatorname{deg} \phi_{i}\right) t^{i} \phi_{i}
$$

corresponding to the Euler vector field (5). If Formal Semisimplicity (24) holds, then we have the decomposition

$$
H_{X} \otimes k=\bigoplus_{i=1}^{N} k \delta_{i}, \quad \delta_{i} * \delta_{j}= \begin{cases}\delta_{i} & \text { if } i=j, \\ 0 & \text { otherwise }\end{cases}
$$

and $(E *)$ is a semisimple operator with eigenvalues $u^{0}, \ldots, u^{N} \in k$ such that $E * \delta_{i}=u_{i} \delta_{i}$. We define $\Delta^{i} \in k$ by

$$
\Delta^{i}=\frac{1}{g\left(\delta_{i}, \delta_{i}\right)},
$$

where $g$ stands for the Poincaré pairing. Then, as in Proposition 5.1, the differential equation

$$
\left(z \frac{\partial}{\partial z}+\frac{1}{z} E *+\mu\right) S=0
$$

has a unique solution of the form $S=\Psi R e^{U / z}$ such that:

(1) $\Psi \in \operatorname{Hom}\left(k^{N+1}, H_{X} \otimes k\right)$ sends the $i$ th standard basis vector in $k^{N+1}$ to the $i$ th normalized idempotent $\sqrt{\Delta^{i}} \delta_{i}$,

(2) $R \in \operatorname{End}\left(k^{N+1}, k^{N+1}\right) \llbracket z \rrbracket$ with $R \equiv \mathrm{Id} \bmod z$,

(3) $U=\operatorname{diag}\left(u^{0}, \ldots, u^{N}\right)$.

Hence, we can define the abstract ancestor potential as

$$
\mathcal{A}_{\text {formal }}^{\text {abs }}=e^{-(1 / 48) \sum_{i} \log \Delta^{i}} \widehat{\Psi} \widehat{R}(\mathcal{T})
$$

(cf. Definitions 4.2 and 5.2). $\mathcal{A}_{\text {formal }}^{\text {abs }}$ is a rational element of $\mathfrak{F o c k}\left(H_{X} \otimes k, \phi_{0}\right)$ with discriminant $\operatorname{det}\left(-q_{1} *\right)$. We will further see that it arises from a formal Frobenius manifold over $k$ as the ancestor potential at the origin. 
THEOREM 6.4 (Teleman [36]). Let $X$ be a smooth projective variety such that Formal Semisimplicity (24) holds. Recall the definition of the total ancestor potential $\mathcal{A}_{X}$ in equation (10) and the definition of the ring $\Upsilon$ in Remark 3.12. We have:

$$
\mathcal{A}_{X}=\mathcal{A}_{\text {formal }}^{\text {abs }} \text {. }
$$

In particular, $\mathcal{A}_{X}$ is a rational element of $\mathfrak{F o c k}\left(H_{X} \otimes \Upsilon, \phi_{0}\right)$ with discriminant $\operatorname{det}\left(-q_{1} *\right)$.

Proof. This is a direct consequence of Teleman's result. We spell out how the Gromov-Witten theory of $X$ defines both a Deligne-Mumford field theory (DMT) over $k$ and a formal Frobenius manifold over $k$. This formal Frobenius manifold induces at the origin the data defined before: the Frobenius algebra $\left(H_{X} \otimes k, *, g\right)$ together with $E$ and $\mu$.

Step 1: A DMT over $k$. We first make minor adjustments to the formal setup in Teleman [36]. Recall that a DMT is a family of maps:

$$
Z_{g}^{n}: H_{X}^{\otimes n} \longrightarrow H^{\bullet}\left(\overline{\mathcal{M}}_{g, n}\right), \quad 2 g-2+n>0,
$$

satisfying certain factorization axioms and a vacuum axiom. Pulling back cohomology classes along the maps $\mathrm{ev}_{i}: X_{g, n, d} \rightarrow X$, capping with the virtual fundamental class, and then pushing forward along the canonical map $X_{g, n, d} \rightarrow \overline{\mathcal{M}}_{g, n}$ defines maps

$$
G W_{g, d}^{n}: H_{X}^{\otimes n} \longrightarrow H^{\bullet}\left(\overline{\mathcal{M}}_{g, n}\right), \quad 2 g-2+n>0,
$$

and setting

$$
Z_{g}^{n}=\sum_{d \in \mathrm{NE}(X)} G W_{g, d}^{n} Q^{d}
$$

defines a DMT over $\Lambda$. Let $t \in H_{X}$ be $t=t^{0} \phi_{0}+\cdots+t^{N} \phi_{N}$ as before. Setting

$$
\begin{aligned}
& { }_{t} Z_{g}^{n}\left(x_{1}, \ldots, x_{n}\right) \\
& \quad=\sum_{m \geq 0} \frac{1}{m !} \int_{\overline{\mathcal{M}}_{g, n+m}}^{\overline{\mathcal{M}}_{g, n}} Z_{g}^{n+m}\left(x_{1}, \ldots, x_{n}, t, \ldots, t\right), \quad 2 g-2+n>0,
\end{aligned}
$$

where the integral denotes the push-forward along the canonical map $\overline{\mathcal{M}}_{g, n+m} \rightarrow$ $\overline{\mathcal{M}}_{g, n}$, defines a formal family of DMTs over $\Lambda$, parameterized by Spf $\Lambda \llbracket t \rrbracket ;$ cf. [36, Sect. 7]. We regard this as a single DMT over the field $k$.

Step 2: A formal Frobenius manifold over $k$. We now deform this DMT to construct a family of DMTs parameterized by $\operatorname{Spf} k \llbracket s^{0}, \ldots, s^{N} \rrbracket$ and hence a formal Frobenius manifold over $k$. (The genus-zero part of any DMT is a tree-level cohomological field theory in the sense of [33, Chap. III, Sect. 4] and thus determines a formal Frobenius manifold.) Define

$$
\begin{aligned}
& { }_{s, t} Z_{g}^{n}\left(x_{1}, \ldots, x_{n}\right) \\
& \quad=\sum_{m \geq 0} \frac{1}{m !} \int_{\overline{\mathcal{M}}_{g, n+m}}^{\overline{\mathcal{M}}_{g, n}}{ }_{t} Z_{g}^{n+m}\left(x_{1}, \ldots, x_{n}, s, \ldots, s\right), \quad 2 g-2+n>0,
\end{aligned}
$$


where $s \in H_{X}$ is $s=s^{0} \phi_{0}+\cdots+s^{N} \phi_{N}$. As in [36, Sect. 7], this defines a family of DMTs over $k$, parameterized by $\operatorname{Spf} k \llbracket s^{0}, \ldots, s^{N} \rrbracket$. It is easy to check that this family is homogeneous ${ }^{5}$ of weight $D=\operatorname{dim}_{\mathbb{C}} X$ with respect to the Euler vector field $\mathcal{E}$ on $\operatorname{Spf} k \llbracket s^{0}, \ldots, s^{N} \rrbracket$ :

$$
\mathcal{E}=\rho^{1} \frac{\partial}{\partial s^{1}}+\cdots+\rho^{r} \frac{\partial}{\partial s^{r}}+\sum_{i=0}^{i=N}\left(1-\frac{\operatorname{deg} \phi_{i}}{2}\right)\left(s^{i}+t^{i}\right) \frac{\partial}{\partial s^{i}},
$$

where $c_{1}(X)=\rho^{1} \phi_{1}+\cdots+\rho^{r} \phi_{r}$; note the shift compared to the Euler field in equation (5). The formal Frobenius manifold over $k$ defined by the DMT is therefore conformal with Euler vector field $\mathcal{E}$. The Euler vector field $\mathcal{E}$ induces the element (27) at the origin and defines the grading operator $\mu$ by

$$
\mu=\left(1-\frac{D}{2}\right) \mathrm{Id}-\nabla^{\mathrm{LC}} \mathcal{E} .
$$

Formal Semisimplicity (24) guarantees that this formal Frobenius manifold induces a semisimple Frobenius algebra $\left(H_{X} \otimes k, *, g\right)$ at the origin.

Step 3: Applying Teleman's Theorem. Teleman's theorem now implies that the ancestor potential for the family of DMTs constructed in Step 2 coincides with the abstract ancestor potential for the formal Frobenius manifold constructed in Step 2. On setting $s=0$, the ancestor potential for the family of DMTs becomes the geometrically defined ancestor potential $\mathcal{A}_{X}$ (see equation (10)). Thus,

$$
\mathcal{A}_{X}=\mathcal{A}_{\text {formal }}^{\text {abs }} \text {. }
$$

The right-hand side here is a priori a formal power series in the variables $y_{j}^{\beta}$ with coefficients in $k$, but since it coincides with the left-hand side, we know from Remark 3.12 that it is in fact a formal power series in the variables $y_{j}^{\beta}$ with coefficients in $\Upsilon$. Moreover, $\mathcal{A}_{\text {formal }}^{\text {abs }}$ is rational over $k$ with discriminant $\operatorname{det}\left(-q_{1} *\right)$; this implies that $\mathcal{A}_{X}$ is rational over $\Upsilon$ with $\operatorname{discriminant} \operatorname{det}\left(-q_{1} *\right)$.

\subsection{Convergence of the Total Ancestor Potential}

Theorem 6.5. Let $X$ be a smooth projective variety such that Genus-Zero Convergence (25) and Analytic Semisimplicity (26) hold. The total ancestor potential $\mathcal{A}_{X}$ is convergent in the sense of Definition 3.13; more precisely, $\mathcal{A}_{X}$ is a rational element of $\mathfrak{F o c k}\left(H_{X} \otimes \Upsilon_{\epsilon}, \phi_{0}\right)$ for some $\epsilon>0$, with discriminant $\operatorname{det}\left(-q_{1} *\right)$. Moreover, we have

$$
\left.\mathcal{A}_{X}\right|_{Q_{1}=\cdots=Q_{r}=1}=\mathcal{A}_{\mathrm{an}}^{\mathrm{abs}} .
$$

Proof. Let Frac denote the fraction field and overline denote the algebraic closure, so that

$$
k=\overline{\operatorname{Frac} \Lambda \llbracket t \rrbracket} .
$$

${ }^{5}$ See [36, Def. 7.16]. 
Let

$$
\begin{aligned}
& k_{1}=\overline{\operatorname{Frac} \mathbb{Q} \llbracket t^{0}, Q_{1} e^{t^{1}}, \ldots, Q_{r} e^{t^{r}}, t^{r+1}, \ldots, t^{N} \rrbracket}, \\
& k_{2}=\overline{\operatorname{Frac} \mathbb{Q} \llbracket t^{0}, e^{t^{1}}, \ldots, e^{t^{r}}, t^{r+1}, \ldots, t^{N} \rrbracket}, \\
& k_{3}=\overline{\operatorname{Frac} \mathbb{Q}\left\{t^{0}, e^{t^{1}}, \ldots, e^{t^{r}}, t^{r+1}, \ldots, t^{N}\right\}}, \\
& k_{4}=\mathbb{Q} \llbracket t^{0}, e^{t^{1}}, \ldots, e^{t^{r}}, t^{r+1}, \ldots, t^{N} \rrbracket, \\
& k_{5}=\mathbb{Q}\left\{t^{0}, e^{t^{1}}, \ldots, e^{t^{r}}, t^{r+1}, \ldots, t^{N}\right\} .
\end{aligned}
$$

Lemma 6.6 will show that $k_{3} \cap k_{4}=k_{5}$.

The divisor equation implies that all of the ingredients $\Delta^{i}, \Psi$, and $R$ used to define $\mathcal{A}_{\text {formal }}^{\text {abs }}$ (in Sect. 6.1) are defined over $k_{1}$ and therefore that $\mathcal{A}_{\text {formal }}^{\text {abs }}$ is an element of $\mathfrak{F o c k}\left(H_{X} \otimes k_{1}, \phi_{0}\right)$. The specialization $Q_{1}=\cdots=Q_{r}=1$ defines an isomorphism $k_{1} \cong k_{2}$, and thus $\mathcal{A}_{\text {formal }}^{\text {abs }} \mid Q_{1}=\cdots=Q_{r}=1$ is a well-defined element of $\mathfrak{F o c k}\left(H_{X} \otimes k_{2}, \phi_{0}\right)$.

On the other hand, all of the ingredients $\Delta^{i}(t), \Psi_{t}$, and $R_{t}$ used to define $\mathcal{A}_{\text {an }}^{\text {abs }}$ (in Sect. 4) are defined over $k_{3}$, and therefore $\mathcal{A}_{\text {an }}^{\text {abs }}$ is an element of $\mathfrak{F o c k}\left(H_{X} \otimes k_{3}, \phi_{0}\right)$. Note that $k_{3}$ is contained in $k_{2}$. Because the two sets of ingredients $\left(\Delta^{i}(t), \Psi_{t}, R_{t}\right)$ and $\left(\Delta^{i}, \Psi, R\right)$ coincide under the maps between ground fields $k_{3} \rightarrow k_{2}$ and $k_{1} \rightarrow k_{2}$, it follows that

$$
\mathcal{A}_{\mathrm{an}}^{\mathrm{abs}}=\left.\mathcal{A}_{\text {formal }}^{\mathrm{abs}}\right|_{Q_{1}=\cdots=Q_{r}=1}
$$

as elements of $\mathfrak{F o c k}\left(H_{X} \otimes k_{2}, \phi_{0}\right)$.

By Theorem 6.4 the right-hand side of (28) equals $\left.\mathcal{A}_{X}\right|_{Q_{1}=\cdots=Q_{r}=1}$ and is an element of $\mathfrak{F} \mathfrak{o c k}\left(H_{X} \otimes k_{4}, \phi_{0}\right)$. Note that $k_{4}$ is contained in $k_{2}$. Since the left-hand side of (28) is defined over $k_{3} \subset k_{2}$, it follows that $\mathcal{A}_{\text {an }}^{\text {abs }}, \mathcal{A}_{\text {formal }}^{\text {abs }} \mid Q_{1}=\cdots=Q_{r}=1$, and $\left.\mathcal{A}_{X}\right|_{Q_{1}=\cdots=Q_{r}=1}$ (which are all equal) are all defined over $k_{3} \cap k_{4}=k_{5}$, that is, all three are elements of $\mathfrak{F o c k}\left(H_{X} \otimes k_{5}, \phi_{0}\right)$.

Because $\mathcal{M}$ is a neighborhood (4) of the large-radius limit point, it contains the set

$$
\left\{\left(t^{0}, \ldots, t^{N}\right) \mid\left(t^{0}, e^{t^{1}}, \ldots, e^{t^{r}}, t^{r+1}, \ldots, t^{N}\right) \in B_{\epsilon}\right\}
$$

for some $\epsilon>0$, where $B_{\epsilon}=\left\{\left(z_{0}, \ldots, z_{N}\right) \in \mathbb{C}^{N+1}|| z_{i} \mid<\epsilon\right\}$. By Remark 4.4, $\mathcal{A}_{\mathrm{an}}^{\mathrm{abs}}$ is also an element of $\mathfrak{F o c k}\left(H_{X} \otimes \mathcal{O}\left(B_{\epsilon}^{\mathrm{ss}}\right), \phi_{0}\right)$ where $B_{\epsilon}^{\mathrm{ss}} \subset B_{\epsilon} \cap(\mathbb{C} \times$ $\left.\left(\mathbb{C}^{\times}\right)^{r} \times \mathbb{C}^{N-r}\right)$ denotes the semisimple locus. Therefore, when expanding $\log \mathcal{A}_{\text {an }}^{\text {abs }}$ in variables $y_{j}^{\beta}$ and $\hbar$, each coefficient is analytic function on $B_{\epsilon}^{\text {ss }}$ that extends to a neighborhood of the origin in $B_{\epsilon}$. Observe that $Z=B_{\epsilon} \backslash B_{\epsilon}^{\mathrm{ss}}$ is a locally finite union of irreducible analytic subvarieties. Thus, there exists $\epsilon^{\prime}$ such that $0<\epsilon^{\prime}<\epsilon$ and that $B_{\epsilon^{\prime}}$ does not meet any irreducible component of $Z$ that is away from the origin. Every coefficient (of the expansion of $\log \mathcal{A}_{\text {an }}^{\text {abs }}$ in variables $y_{j}^{\beta}$ and $\hbar$ ) extends to a holomorphic function on $B_{\epsilon^{\prime}}$. This shows that $\left.\mathcal{A}_{X}\right|_{Q_{1}=\ldots=Q_{r}=1}$ is convergent in the sense of Definition 3.13 or, in other words,

$$
\left.\mathcal{A}_{X}\right|_{Q_{1}=\cdots=Q_{r}=1} \in \mathfrak{F o c k}\left(H_{X} \otimes \Upsilon_{\epsilon^{\prime}}, \phi_{0}\right) .
$$


Finally, the rationality of $\left.\mathcal{A}_{X}\right|_{Q_{1}=\cdots=Q_{r}=1}$ follows from the rationality of $\mathcal{A}_{\text {an }}^{\text {abs }}$ and the fact that the $\operatorname{discriminant} \operatorname{det}\left(-q_{1} *\right)$ is an element of $\Upsilon_{\epsilon^{\prime}}\left[q_{1}^{0}, \ldots, q_{1}^{N}\right]$.

LemMa 6.6. Consider the intersections

$$
\begin{aligned}
& \overline{\text { Frac } \mathbb{C}\left\{x_{1}, \ldots, x_{n}\right\}} \cap \mathbb{C} \llbracket x_{1}, \ldots, x_{n} \rrbracket \subset \overline{\text { Frac } \mathbb{C} \llbracket x_{1}, \ldots, x_{n} \rrbracket}, \\
& \overline{\text { Frac } \mathbb{Q}\left\{x_{1}, \ldots, x_{n}\right\}} \cap \mathbb{Q} \llbracket x_{1}, \ldots, x_{n} \rrbracket \subset \overline{\text { Frac } \mathbb{Q} \llbracket x_{1}, \ldots, x_{n} \rrbracket} .
\end{aligned}
$$

We have:

1. $\overline{\text { Frac } \mathbb{C}\left\{x_{1}, \ldots, x_{n}\right\}} \cap \mathbb{C} \llbracket x_{1}, \ldots, x_{n} \rrbracket=\mathbb{C}\left\{x_{1}, \ldots, x_{n}\right\}$,

2. $\overline{\text { Frac } \mathbb{Q}\left\{x_{1}, \ldots, x_{n}\right\}} \cap \mathbb{Q} \llbracket x_{1}, \ldots, x_{n} \rrbracket=\mathbb{Q}\left\{x_{1}, \ldots, x_{n}\right\}$.

Proof. Statement (1) immediately implies statement (2). We prove (1). Let $P\left(x_{1}, \ldots, x_{n}, y\right)=f_{0}\left(x_{1}, \ldots, x_{n}\right) y^{k}+f_{1}\left(x_{1}, \ldots, x_{n}\right) y^{k-1}+\cdots+f_{k}\left(x_{1}, \ldots, x_{n}\right)$, where $f_{i} \in \mathbb{C}\left\{x_{1}, \ldots, x_{n}\right\}$. Assume that the equation $P\left(x_{1}, \ldots, x_{n}, y\right)=0$ has a solution $y=g\left(x_{1}, \ldots, x_{n}\right)$ with $g \in \mathbb{C} \llbracket x_{1}, \ldots, x_{n} \rrbracket$, so that

$$
P\left(x_{1}, \ldots, x_{n}, g\left(x_{1}, \ldots, x_{n}\right)\right)=0 .
$$

We will show that $g \in \mathbb{C}\left\{x_{1}, \ldots, x_{n}\right\}$. Without loss of generality we may assume that $g(0, \ldots, 0)=0$ and therefore that $P(0,0, \ldots, 0,0)=0$.

Suppose first that $P(0,0, \ldots, 0, y)$ is not identically zero. Then the Weierstrass preparation theorem implies that

$$
P\left(x_{1}, \ldots, x_{n}, y\right)=W\left(x_{1}, \ldots, x_{n}, y\right) h\left(x_{1}, \ldots, x_{n}, y\right),
$$

where $h$ is a unit in the local ring at the origin and $W$ is a Weierstrass polynomial:

$$
W\left(x_{1}, \ldots, x_{n}, y\right)=y^{l}+\sum_{j=0}^{l-1} w_{j}\left(x_{1}, \ldots, x_{n}\right) y^{j}
$$

with $w_{j}(0, \ldots, 0)=0$. Then $W\left(x_{1}, \ldots, x_{n}, g\left(x_{1}, \ldots, x_{n}\right)\right)=0$. A theorem of Aroca [2] implies that there exist vectors

$$
v_{1}, \ldots, v_{n} \in \mathbb{Q}^{n}, \quad v_{i}=\left(v_{i}^{1}, \ldots, v_{i}^{n}\right),
$$

such that $v_{1}, \ldots, v_{n}$ span a strictly convex cone containing the positive orthant, that the $\mathbb{Z}_{\geq 0}$-span of $v_{1}, \ldots, v_{n}$ contains $\left(\mathbb{Z}_{\geq 0}\right)^{n}$, and that after the monomial change of variables,

$$
z_{i}=x_{1}^{v_{i}^{1}} \cdots x_{n}^{v_{i}^{n}}, \quad i=1,2, \ldots, n,
$$

there exists a convergent power series $y_{c} \in \mathbb{C}\left\{z_{1}, \ldots, z_{n}\right\}$ such that

$$
W\left(x_{1}, \ldots, x_{n}, y_{c}\left(z_{1}, \ldots, z_{n}\right)\right)=0 .
$$

We can therefore factorize $W$ over the ring $\mathbb{C}\left\{z_{1}, \ldots, z_{n}\right\}$ :

$$
W\left(x_{1}, \ldots, x_{n}, y\right)=\left(y-y_{c}\right)\left(y^{l-1}+\sum_{j=0}^{l-2} w_{j}^{\prime}\left(z_{1}, \ldots, z_{n}\right) y^{j}\right) .
$$


This equation makes sense over the ring $\mathbb{C} \llbracket z_{1}, \ldots, z_{n} \rrbracket$ that contains the solution $y=g\left(x_{1}, \ldots, x_{n}\right)$. Thus, either $y_{c}=g$, in which case $g \in \mathbb{C}\left\{x_{1}, \ldots, x_{n}\right\}$, or we can apply Aroca's theorem again with $W\left(x_{1}, \ldots, x_{n}, y\right)$ replaced by the Weierstrass polynomial

$$
y^{l-1}+\sum_{j=0}^{l-2} w_{j}^{\prime}\left(z_{1}, \ldots, z_{n}\right) y^{j}
$$

of lower degree. By induction, we conclude that $g \in \mathbb{C}\left\{x_{1}, \ldots, x_{n}\right\}$.

It remains to consider the case where $P(0,0, \ldots, 0, y)$ is identically zero. Consider the coordinate change

$$
x_{i}^{\prime}=x_{i}-a_{i} y, \quad 1 \leq i \leq n,
$$

where we choose $\left(a_{1}, \ldots, a_{n}\right) \in \mathbb{C}^{n}$ such that $P\left(x_{1}, \ldots, x_{n}, y\right)$ is not identically zero on the line $x_{1}^{\prime}=\cdots=x_{n}^{\prime}=0$ and $d g_{(0,0 \ldots, 0)}\left(a_{1}, \ldots, a_{n}\right) \neq 1$. Writing the solution $y=g\left(x_{1}, \ldots, x_{n}\right)$ in the new coordinate system, we find

$$
y=g\left(x_{1}^{\prime}+a_{1} y, x_{2}^{\prime}+a_{2} y, \ldots, x_{n}^{\prime}+a_{n} y\right) .
$$

This equation has a unique power series solution $y=G\left(x_{1}^{\prime}, \ldots, x_{n}^{\prime}\right)$, and the argument in the preceding paragraph shows that $G \in \mathbb{C}\left\{x_{1}^{\prime}, \ldots, x_{n}^{\prime}\right\}$. To recover $g\left(x_{1}, \ldots, x_{n}\right)$ from $G\left(x_{1}^{\prime}, \ldots, x_{n}^{\prime}\right)$, we solve the equation

$$
y=G\left(x_{1}-a_{1} y, x_{2}-a_{2} y, \ldots, x_{n}-a_{n} y\right) .
$$

This also has a unique power series solution $y=g\left(x_{1}, \ldots, x_{n}\right)$ because the condition $d g_{(0,0 \ldots, 0)}\left(a_{1}, \ldots, a_{n}\right) \neq 1$ implies that $d G_{(0,0 \ldots, 0)}\left(a_{1}, \ldots, a_{n}\right) \neq-1$. On the other hand, the implicit function theorem shows that there is a unique analytic solution $y=v\left(x_{1}, \ldots, x_{n}\right)$ such that $v(0, \ldots, 0)=0$. The power series expansion of $v$ at the origin must coincide with $g\left(x_{1}, \ldots, x_{n}\right)$; thus, $g \in \mathbb{C}\left\{x_{1}, \ldots, x_{n}\right\}$. The lemma is proved.

REMARK 6.7. The same argument proves Givental's statements (A) and (B) for the ancestor potential of a compact toric orbifold. We need:

- the fact that orbifold Gromov-Witten theory defines a DMT (combine [36, Sect. 1.7] with [1]);

- analyticity, semisimplicity, and tameness of the corresponding Frobenius manifold.

This last point would follow from an appropriate mirror theorem for toric orbifolds. Such a mirror theorem has been formulated as the Coates-Corti-IritaniTseng conjecture (see [26, Sect. 4]), which was proved for weighted projective spaces in [8] and will be proved for general toric orbifolds $X$ in [7].

Tseng has announced a proof of statements (A) and (B) for compact toric orbifolds using localization in equivariant Gromov-Witten theory [34]. His version is somewhat stronger than ours since it applies in the equivariant setting where the Frobenius manifold is not conformal. 


\section{NF-Convergence of Gromov-Witten Potentials: Statements}

Definition 7.1. The genus- $g$ ancestor potential $\overline{\mathcal{F}}_{t}^{g}$ is said to be $N F$-convergent if the power series (9) converges absolutely and uniformly on an infinitedimensional polydisc of the form

$$
\begin{cases}\left|y_{i}^{\alpha}\right|<\epsilon i ! / C^{i}, & 0 \leq i<\infty, 0 \leq \alpha \leq N \\ \left|t^{\alpha}\right|<\epsilon, & 0 \leq \alpha \leq N, \\ \left|Q_{j}\right|<\epsilon, & 1 \leq j \leq r,\end{cases}
$$

for some $C, \epsilon>0$. The total ancestor potential $\mathcal{A}_{X}$ is said to be $N F$-convergent if the power series (9) defining each genus- $g$ ancestor potential $\overline{\mathcal{F}}_{t}^{g}$ converges absolutely and uniformly on a polydisc of the form (29) for some uniform $C, \epsilon>0$.

REMARK 7.2. "NF” here stands for "nuclear Fréchet": see Remark 7.6.

THEOREM 7.3. If the total ancestor potential $\mathcal{A}_{X}$ is convergent in the sense of Definition 3.13, then it is NF-convergent in the sense of Definition 7.1.

REMARK 7.4. NF-convergence of the total ancestor potential (Def. 7.1) is weaker than convergence of the total ancestor potential (Def. 3.13). The rationality and the tameness in Definition 3.13 do not follow from NF-convergence.

Theorem 6.5 and Theorem 7.3 together immediately imply Theorem 1.4.

\subsection{Convergence of the Descendant Potential}

Definition 7.5. The genus- $g$ descendant Gromov-Witten potential $\mathcal{F}_{X}^{g}$ is said to be $N F$-convergent if the power series (6) converges absolutely and uniformly on an infinite-dimensional polydisc of the form

$$
\begin{cases}\left|t_{i}^{\alpha}\right|<\epsilon i ! / C^{i}, & 0 \leq i<\infty, 0 \leq \alpha \leq N \\ \left|Q_{j}\right|<\epsilon, & 1 \leq j \leq r\end{cases}
$$

for some $C, \epsilon>0$. We say that the total descendant Gromov-Witten potential $\mathcal{Z}_{X}$ is $N F$-convergent if the power series (6) defining each genus- $g$ descendant potential $\mathcal{F}_{X}^{g}$ converges absolutely and uniformly on a polydisc of the form (30) for some uniform $C, \epsilon>0$.

REMARK 7.6. A holomorphic function on a locally convex topological vector space over $\mathbb{C}$ can be defined as a complex Gâteaux-differentiable continuous function $[12 ; 5]$. If $\mathcal{F}_{X}^{g}$ is NF-convergent, then it defines a holomorphic function on an $\epsilon$-ball of the Banach space

$$
l_{\infty}^{C}\left(H_{X}\right)=\left\{\mathbf{t}(z) \in H_{X} \otimes \mathbb{C} \llbracket z \rrbracket: \sup _{i, \alpha}\left(\frac{\left|t_{i}^{\alpha}\right| C^{i}}{i !}\right)<\infty\right\}
$$


equipped with the weighted $l_{\infty}$-norm

$$
\|\mathbf{t}\|_{\infty, \log C}=\sup _{i, \alpha}\left(\frac{\left|t_{i}^{\alpha}\right| C^{i}}{i !}\right) .
$$

If $\mathcal{F}_{X}^{g}$ is NF-convergent, then we can also view it as a holomorphic function on a neighborhood of the origin of the nuclear Fréchet space

$\mathcal{H}_{+}=\left\{\mathbf{t}(z) \in H_{X} \otimes \mathbb{C} \llbracket z \rrbracket: \sup _{i, \alpha}\left(\frac{\left|t_{i}^{\alpha}\right| e^{i n}}{i !}\right)<\infty\right.$ for all $\left.n \geq 0\right\} \subset l_{\infty}^{C}\left(H_{X}\right)$.

The topology on $\mathcal{H}_{+}$is defined by countably many norms

$$
\|\mathbf{t}\|_{\infty, n}=\sup _{i, \alpha}\left(\frac{\left|t_{i}^{\alpha}\right| e^{n i}}{i !}\right), \quad n=0,1,2, \ldots
$$

This viewpoint is perhaps more natural. As we will see in Lemma 8.9, a holomorphic function on a neighborhood of zero in $\mathcal{H}_{+}$automatically extends to a holomorphic function on a neighborhood of zero in $l_{\infty}^{C}\left(H_{X}\right)$ for some $C>0$.

REMARK 7.7. In unpublished work, Iritani has shown that the Gromov-Witten potential $\mathcal{F}_{X}^{g}$ converges on a polydisc of the form (30) whenever the target space $X$ admits a torus action with isolated fixed points and isolated one-dimensional orbits [27].

THEOREM 7.8. If the nondescendant genus-zero potential $F_{X}^{0}$ is convergent in the sense of Section 2.3, then the genus-zero descendant potential $\mathcal{F}_{X}^{0}$ is $N F$ convergent in the sense of Definition 7.5.

THEOREM 7.9. If the total ancestor potential $\mathcal{A}_{X}$ is convergent in the sense of Definition 3.13, then the total descendant potential $\mathcal{Z}_{X}$ is NF-convergent in the sense of Definition 7.5 .

Theorem 6.5 and Theorem 7.9 together immediately imply Theorem 1.1.

\section{NF-Convergence of Gromov-Witten Potentials: Proofs}

In this section we prove the results about NF-convergence of descendant and ancestor potentials stated in Section 7. The key ingredients are the KontsevichManin ancestor-descendant relation, the Nash-Moser inverse function theorem, and a version of Givental's symplectic space based on a nuclear Fréchet space (see Sect. 8.4), which may be of independent interest.

\subsection{Setting $Q_{1}=\cdots=Q_{r}=1$ Makes Sense when $\mathcal{F}_{X}^{g}$ is NF-Convergent}

Making the argument explicit, we write the genus- $g$ descendant potential $\mathcal{F}_{X}^{g}$ as

$$
\mathcal{F}_{X}^{g}\left(\mathbf{q}, Q_{1}, \ldots, Q_{r}\right),
$$


where $\mathbf{q}$ is the dilaton-shifted coordinate appearing in Section 2.6 and $Q_{1}, \ldots, Q_{r}$ are Novikov variables. The divisor equation [1, Thm. 8.3.1] implies that

$$
\begin{aligned}
\mathcal{F}_{X}^{g}\left(\left[e^{-\delta / z} \mathbf{q}(z)\right]_{+}, Q_{1}, \ldots, Q_{r}\right)= & \mathcal{F}_{X}^{g}\left(\mathbf{q}(z), e^{\delta_{1}} Q_{1}, \ldots, e^{\delta_{r}} Q_{r}\right) \\
& +\frac{\delta_{g, 0}}{2} \Omega\left(e^{-\delta / z} \mathbf{q}(z),\left[e^{-\delta / z} \mathbf{q}(z)\right]_{+}\right) \\
& -\frac{\delta_{g, 1}}{24} \int_{X} \delta \cup c_{D-1}(X),
\end{aligned}
$$

where $\delta=\sum_{\alpha=1}^{r} \delta_{\alpha} \phi_{\alpha} \in H^{2}(X), D=\operatorname{dim} X,[\cdots]_{+}$denotes the power series truncation of a Laurent series in $z$, and $\Omega$ is Givental's symplectic form in (18) (with $V=H_{X}$ ). The formula follows by integrating [9, Eq. (8)] and using [19, Prop. 5.3]. Equation (34) is an equality between formal power series in the variables $t_{i}^{\alpha}, Q_{i}$, and $\delta_{\alpha}$, where

$$
t_{i}^{\alpha}= \begin{cases}q_{i}^{\alpha}+1 & \text { if }(i, \alpha)=(1,0), \\ q_{i}^{\alpha} & \text { otherwise. }\end{cases}
$$

Note that the specialization $Q_{1}=\cdots=Q_{r}=1$ of the right-hand side of (34) makes sense as an analytic function on a region $\left\{\left(\mathbf{q}(z)=\mathbf{t}(z)-\phi_{0} z, \delta\right)\right.$ : $\left.\|\mathbf{t}\|_{\infty, \log C}<\epsilon,\left|e^{\delta_{\alpha}}\right|<\epsilon\right\} \subset l_{\infty}^{C}\left(H_{X}\right) \times H^{2}(X ; \mathbb{C})$ if $\mathcal{F}_{X}^{g}$ is NF-convergent (see (31), (32) for the Banach space $\left.l_{\infty}^{C}\left(H_{X}\right)\right)$.

Lemma 8.1. Assume that the genus-g descendant potential $\mathcal{F}^{g}$ is NF-convergent in the sense of Definition 7.5. Then the specialization $Q_{1}=\cdots=Q_{r}=1$ of the right-hand side of (34) depends only on the point $\left[e^{-\delta / z} \mathbf{q}(z)\right]_{+} \in H_{X} \otimes \mathbb{C} \llbracket z \rrbracket$.

Proof. Suppose $\left[e^{-\delta / z} \mathbf{q}(z)\right]_{+}=\left[e^{-\delta^{\prime} / z} \mathbf{q}^{\prime}(z)\right]_{+}$. We need to show that the specialization $Q_{1}=\cdots=Q_{r}=1$ of the right-hand side of (34) has the same value at $(\mathbf{q}, \delta)$ and $\left(\mathbf{q}^{\prime}, \delta^{\prime}\right)$. This follows by applying (34) itself to the relation $\mathbf{q}^{\prime}(z)=\left[e^{\left(\delta^{\prime}-\delta\right) / z} \mathbf{q}(z)\right]_{+}$.

The lemma allows us to define a holomorphic function $\mathcal{F}_{X \text {,an }}^{g}$ as follows.

Definition-Proposition 8.2. Assume that the genus- $g$ descendant potential $\mathcal{F}^{g}$ is NF-convergent in the sense of Definition 7.5. Recall the definition of the Banach space $l_{\infty}^{C}\left(H_{X}\right)$ in Remark 7.6 and set

$$
B_{\epsilon}\left(l_{\infty}^{C}\left(H_{X}\right)\right)=\left\{\mathbf{t}(z) \in l_{\infty}^{C}\left(H_{X}\right):\|\mathbf{t}\|_{\infty, C}<\epsilon\right\} .
$$

Then there exists a holomorphic function

$$
\mathcal{F}_{X, \text { an }}^{g}: \bigcup_{\substack{\delta \in H^{2}(X ; \mathbb{C}), \Re\left(\delta_{i}\right)<\log \epsilon}}\left[e^{-\delta / z}\left(-\phi_{0} z+B_{\epsilon}\left(l_{\infty}^{C}\left(H_{X}\right)\right)\right)\right]_{+} \rightarrow \mathbb{C}
$$


such that

$$
\begin{aligned}
\mathcal{F}_{X, \text { an }}^{g}\left(\left[e^{-\delta / z} \mathbf{q}(z)\right]_{+}\right)= & \mathcal{F}_{X}^{g}\left(\mathbf{q}, e^{\delta_{1}}, \ldots, e^{\delta_{r}}\right) \\
& +\frac{\delta_{g, 0}}{2} \Omega\left(e^{-\delta / z} \mathbf{q}(z),\left[e^{-\delta / z} \mathbf{q}(z)\right]_{+}\right) \\
& -\frac{\delta_{g, 1}}{24} \int_{X} \delta \cup c_{D-1}(X) .
\end{aligned}
$$

We refer to $\mathcal{F}_{X, \text { an }}^{g}$ as the specialization of $\mathcal{F}_{X}^{g}$ to $Q_{1}=\cdots=Q_{r}=1$.

\subsection{Fundamental Solution}

Recall the definition of the Dubrovin connection $\nabla$ in Section 2.4. Consider the fundamental solution $L \in \operatorname{End}\left(H_{X}\right) \otimes \Lambda \llbracket t \rrbracket \llbracket z^{-1} \rrbracket$ defined by

$$
L(t, z) v=v+\sum_{d \in \operatorname{NE}(X)} \sum_{n=0}^{\infty} \sum_{\epsilon=0}^{N} \frac{Q^{d}}{n !}\left\langle\frac{v}{z-\psi}, t, \ldots, t,\left.\phi^{\epsilon}\right|_{0, n+2, d} ^{X} \phi_{\epsilon}\right.
$$

for $v \in H_{X}$. The expression $v /(z-\psi)$ in the correlator should be expanded in the series $\sum_{n=0}^{\infty} v \psi^{n} z^{-n-1}$. The fundamental solution satisfies

$$
\begin{aligned}
& \nabla_{\partial / \partial t^{i}}\left(L(t, z) z^{-\mu} z^{-\rho} v\right)=0 \quad \text { and } \\
& \nabla_{z \partial / \partial z}\left(L(t, z) z^{-\mu} z^{-\rho} v\right)=0
\end{aligned}
$$

for all $v \in H_{X}$, where $\rho=c_{1}(X)$, and the endomorphisms $z^{-\mu}$ and $z^{-\rho}$ of $H_{X}$ are defined by $z^{-\mu}=\exp (-\mu \log z)$ and $z^{-\rho}=\exp (-\rho \log z)$. The fundamental solution also satisfies

$$
(L(t,-z) v, L(t, z) w)=(v, w)
$$

for $v, w \in H_{X}$, where $(\cdot, \cdot)$ denotes the Poincaré pairing of $H_{X}$, and so the inverse fundamental solution $M(t, z)=L(t, z)^{-1}$ coincides with the adjoint of $L(t,-z)$ :

$$
M(t, z) v:=v+\sum_{d \in \operatorname{NE}(X)} \sum_{n=0}^{\infty} \sum_{\epsilon=0}^{N} \frac{Q^{d}}{n !}\left\langle\frac{\phi^{\epsilon}}{-z-\psi}, t, \ldots, t, v\right\rangle_{0, n+2, d}^{X} \phi_{\epsilon} .
$$

The divisor equation for descendant invariants [1, Thm. 8.3.1] implies that

$$
L(t, z) v=e^{\delta / z} v+\sum_{d \in \operatorname{NE}(X)} \sum_{n=0}^{\infty} \sum_{\epsilon=0}^{N} \frac{e^{d \cdot \delta} Q^{d}}{n !}\left\langle\frac{e^{\delta / z} v}{z-\psi}, t^{\prime}, \ldots, t^{\prime},\left.\phi^{\epsilon}\right|_{0, n+2, d} ^{X} \phi_{\epsilon},\right.
$$

where $t=\delta+t^{\prime}, \delta \in H^{2}(X), t^{\prime} \in \bigoplus_{p \neq 1} H^{2 p}(X)$.

If the genus-zero Gromov-Witten potential $F_{X}^{0}$ converges in the sense of Section 2.3, then the fundamental solution with $Q_{1}=\cdots=Q_{r}=1$ depends analytically on both $t$ and $z$, where $t$ lies in a neighborhood (4) of the large-radius limit point, and $z$ is any point of $\mathbb{C}^{\times}$. 


\subsection{Ancestor-Descendant Relation}

In this section we distinguish the variables for descendant potentials and ancestor potentials. Let $\mathbf{x}=\left(x_{0}, x_{1}, x_{2}, \ldots\right)$ be a sequence of variables in $H_{X}$ with $x_{i}=$ $\sum_{\alpha=0}^{N} x_{i}^{\alpha} \phi_{\alpha} \in H_{X}$. Let $\mathbf{q}=\left(q_{0}, q_{1}, q_{2}, \ldots\right)$ be a sequence of variables in $H_{X}$ with $q_{i}=\sum_{\alpha=0}^{N} q_{i}^{\alpha} \phi_{\alpha} \in H_{X}$ as before. We consider the change of variables

$$
\mathbf{q}(z)=[M(t, z) \mathbf{x}(z)]_{+}
$$

between $\mathbf{x}$ and $\mathbf{q}$ for some $t \in H_{X}$. Here $[\cdots]_{+}$denotes the truncation of a $z$-series, $\mathbf{x}(z)=\sum_{i=0}^{\infty} x_{i} z^{i}, \mathbf{q}(z)=\sum_{i=0}^{\infty} q_{i} z^{i}$, and $M(t, z)$ is the inverse fundamental solution given in (38). We relate the variables $\mathbf{q}, \mathbf{x}$ with the variables $\mathbf{t}, \mathbf{y}$ by the dilaton shift (cf. Section 2.6):

$$
\mathbf{q}(z)=\mathbf{t}(z)-\phi_{0} z, \quad \mathbf{x}(z)=\mathbf{y}(z)-\phi_{0} z
$$

As in Section 2.5, we use $\mathbf{t}$ as arguments for the descendant potential $\mathcal{F}_{X}^{g}$ and $\mathcal{Z}_{X}$ and use $t, \mathbf{y}$ as arguments for the ancestor potential $\overline{\mathcal{F}}_{X}^{g}$ and $\mathcal{A}_{X}$, that is, $\mathcal{Z}_{X}$ is a formal power series in $\mathbf{t}$, and $\mathcal{A}_{X}$ is a formal power series in $t$ and $\mathbf{y}$.

Theorem 8.3 (Kontsevich-Manin [30, Thm. 2.1], Givental [19, Sect. 5], CoatesGivental [9, App. 2]). Let $\mathcal{F}^{g}$ and $\overline{\mathcal{F}}_{t}^{g}$ denote the genus-g descendant and ancestor potentials of a smooth projective variety $X$. We have:

$$
\mathcal{F}^{g}(\mathbf{q})= \begin{cases}F^{1}(t)+\overline{\mathcal{F}}_{t}^{1}(\mathbf{x}) & \text { if } g=1, \\ \overline{\mathcal{F}}_{t}^{g}(\mathbf{x}) & \text { if } g \geq 2,\end{cases}
$$

under the change of variables given in (40). Here $F^{1}$ is the nondescendant genus1 potential.

REMARK 8.4. In terms of the dilaton-shifted coordinates (41), the change of variables (40) can be written as

$$
\begin{aligned}
& t_{0}=t+y_{0}+M_{1}(t) y_{1}+M_{2}(t) y_{2}+\cdots, \\
& t_{1}=y_{1}+M_{1}(t) y_{2}+M_{2}(t) y_{3}+\cdots, \\
& t_{2}=y_{2}+M_{1}(t) y_{3}+M_{2}(t) y_{4}+\cdots,
\end{aligned}
$$

Here we write $M(t, z)=\mathrm{Id}+\sum_{n=1}^{\infty} M_{n}(t) z^{-n}$ and use $\left[M(t, z)\left(-\phi_{0} z\right)\right]_{+}=$ $-\phi_{0} z+t$. This defines an isomorphism

$$
\Lambda \llbracket \mathbf{t} \rrbracket \llbracket t \rrbracket \stackrel{\cong}{\longrightarrow} \Lambda \llbracket \mathbf{y} \rrbracket \llbracket t \rrbracket
$$

because $M_{n}(0) \equiv 0$ modulo $\left(Q_{1}, \ldots, Q_{r}\right)$ for $n \geq 1$. 


\subsection{Analytic Preliminaries}

Consider the following family of Hilbert norms $\|\cdot\|_{n}, n=0,1,2, \ldots$, on $\mathbb{C} \llbracket z, z^{-1} \rrbracket:$

$$
\|\mathbf{a}(z)\|_{n}=\left(\sum_{j \in \mathbb{Z}} \frac{\left|a_{j}\right|^{2}}{|\Gamma(1 / 2+j)|^{2}} e^{2 n j}\right)^{1 / 2} \text { for } \mathbf{a}(z)=\sum_{j \in \mathbb{Z}} a_{j} z^{j}
$$

and set

$$
\mathbb{C}\left\{\left\{z, z^{-1}\right\}\right\}=\left\{\mathbf{a}(z) \in \mathbb{C} \llbracket z, z^{-1} \rrbracket:\|\mathbf{a}(z)\|_{n}<\infty \text { for all } n \gg 0\right\} .
$$

We write

$$
\begin{aligned}
\mathbb{C}\{\{z\}\} & =\mathbb{C} \llbracket z \rrbracket \cap \mathbb{C}\left\{\left\{z, z^{-1}\right\}\right\}=\left\{\mathbf{a}(z) \in \mathbb{C} \llbracket z \rrbracket:\|\mathbf{a}(z)\|_{n}<\infty \text { for all } n \geq 0\right\}, \\
\mathbb{C}\left\{\left\{z^{-1}\right\}\right\} & =\mathbb{C} \llbracket z^{-1} \rrbracket \cap \mathbb{C}\left\{\left\{z, z^{-1}\right\}\right\} \\
& =\left\{\mathbf{a}(z) \in \mathbb{C} \llbracket z^{-1} \rrbracket:\|\mathbf{a}(z)\|_{n}<\infty \text { for some } n \geq 0\right\} .
\end{aligned}
$$

Note that the norms are increasing on $\mathbb{C}\{\{z\}\}\left(\|\cdot\|_{0} \leq\|\cdot\|_{1} \leq\|\cdot\|_{2} \leq \cdots\right)$, and $\mathbb{C}\{\{z\}\}$ is a nuclear Fréchet space with topology defined by these norms. The norms are decreasing on $\mathbb{C}\left\{\left\{z^{-1}\right\}\right\}\left(\|\cdot\|_{0} \geq\|\cdot\|_{1} \geq\|\cdot\|_{2} \geq \cdots\right)$, and $\mathbb{C}\left\{\left\{z^{-1}\right\}\right\}$ is an inductive limit of Hilbert spaces; $\mathbb{C}\left\{\left\{z^{-1}\right\}\right\}$ with the inductive limit topology is the strong dual of $\mathbb{C}\{\{z\}\}$ and is a nuclear (DF) space. The following lemma shows that $\mathbb{C}\left\{\left\{z, z^{-1}\right\}\right\}$ is a topological ring.

Lemma 8.5. For $\mathbf{a}(z), \mathbf{b}(z) \in \mathbb{C}\left\{\left\{z, z^{-1}\right\}\right\}$, the product $\mathbf{a}(z) \mathbf{b}(z)$ converges. Moreover, we have:

$$
\begin{aligned}
& \left\|[\mathbf{a}(z) \mathbf{b}(z)]_{+}\right\|_{n-1} \leq 5\left(\|\mathbf{a}(z)\|_{n+2}+\|\mathbf{a}(z)\|_{n-2}\right)\left(\|\mathbf{b}(z)\|_{n+2}+\|\mathbf{b}(z)\|_{n-2}\right), \\
& \left\|[\mathbf{a}(z) \mathbf{b}(z)]_{-}\right\|_{n+1} \leq 5\left(\|\mathbf{a}(z)\|_{n+2}+\|\mathbf{a}(z)\|_{n-2}\right)\left(\|\mathbf{b}(z)\|_{n+2}+\|\mathbf{b}(z)\|_{n-2}\right),
\end{aligned}
$$

where $[\cdots]_{+}$and $[\cdots]_{-}$denote respectively the nonnegative and strictly negative truncations of a power series in $z$ and $z^{-1}$. In particular, if $\mathbf{a}(z) \in \mathbb{C}\left\{\left\{z^{-1}\right\}\right\}$ and $\mathbf{b}(z) \in \mathbb{C}\{\{z\}\}$, then:

$$
\begin{aligned}
& \left\|[\mathbf{a}(z) \mathbf{b}(z)]_{+}\right\|_{n-1} \leq 20\|\mathbf{a}(z)\|_{n-2}\|\mathbf{b}(z)\|_{n+2}, \\
& \left\|[\mathbf{a}(z) \mathbf{b}(z)]_{-}\right\|_{n+1} \leq 20\|\mathbf{a}(z)\|_{n-2}\|\mathbf{b}(z)\|_{n+2} .
\end{aligned}
$$

Proof. Observe first that

$$
\left|\frac{\Gamma(j+1 / 2) \Gamma(k+1 / 2)}{\Gamma(j+k+1 / 2)}\right| \leq \pi e^{2|j|+2|k|}
$$

for all $j, k \in \mathbb{Z}$. Setting $\mathbf{c}(z)=\sum_{l \in \mathbb{Z}} c_{l} z^{l}=\mathbf{a}(z) \mathbf{b}(z)$, we have

$$
\begin{aligned}
\left|\frac{c_{l}}{\Gamma(l+1 / 2)}\right| & \leq \sum_{j+k=l} \frac{\left|a_{j} b_{k}\right|}{|\Gamma(l+1 / 2)|} \\
& \leq \sum_{j+k=l} \frac{\left|a_{j}\right|}{|\Gamma(j+1 / 2)|} \frac{\left|b_{k}\right|}{|\Gamma(k+1 / 2)|}\left|\frac{\Gamma(j+1 / 2) \Gamma(k+1 / 2)}{\Gamma(j+k+1 / 2)}\right|
\end{aligned}
$$




$$
\begin{aligned}
& \leq \pi e^{-n l} \sum_{j+k=l} \frac{\left|a_{j}\right|}{|\Gamma(j+1 / 2)|} e^{n j+2|j|} \frac{\left|b_{k}\right|}{|\Gamma(k+1 / 2)|} e^{n k+2|k|} \\
& \leq \pi e^{-n l}\left(\|\mathbf{a}(z)\|_{n+2}+\|\mathbf{a}(z)\|_{n-2}\right)\left(\|\mathbf{b}(z)\|_{n+2}+\|\mathbf{b}(z)\|_{n-2}\right),
\end{aligned}
$$

where we used the Cauchy-Schwarz inequality in the last step. The conclusion follows.

REMARK 8.6. Let $\tau$ be the coordinate Laplace-dual to $z^{-1}$, and let $\left(j_{*} \mathcal{O}_{\mathbb{C}_{\tau}}\right)_{\infty}$ denote the space of germs of holomorphic functions $f$ defined on a small punctured neighborhood

$$
\left\{\tau \in \mathbb{C}: R_{f}<|\tau|<\infty\right\}
$$

of $\tau=\infty$. Here $j: \mathbb{C}_{\tau} \hookrightarrow \mathbb{P}_{\tau}^{1}$ is the natural inclusion. A calculation similar to that in the proof of Lemma 8.5 shows that the ring $\mathbb{C}\left\{\left\{z, z^{-1}\right\}\right\}$ acts on $\left(j_{*} \mathcal{O}_{\mathbb{C}_{\tau}}\right)_{\infty}$ as microdifferential operators

$$
f(\tau) \mapsto \tau^{1 / 2} \mathbf{a}\left(\partial_{\tau}^{-1}\right) \tau^{-1 / 2} f(\tau) \quad \text { for } \mathbf{a}(z) \in \mathbb{C}\left\{\left\{z, z^{-1}\right\}\right\} .
$$

Here $\tau^{-1 / 2}$ was put to make the action well defined. The positive part $\mathbb{C}\{\{z\}\}$ preserves the space of entire functions $\mathcal{O}\left(\mathbb{C}_{\tau}\right) \subset\left(j_{*} \mathcal{O}_{\mathbb{C}_{\tau}}\right)_{\infty}$, and the negative part $\mathbb{C}\left\{\left\{z^{-1}\right\}\right\}$ preserves the space of germs of holomorphic functions $\left(\mathcal{O}_{\mathbb{P}_{\tau}^{1}}\right)_{\infty} \subset$ $\left(j_{*} \mathcal{O}_{\mathbb{C}_{\tau}}\right)_{\infty}$ at $\tau=\infty$.

Definition 8.7 (cf. [21]). We now define a nuclear version of Givental's symplectic space. This is the vector space

$$
\mathcal{H}=H_{X} \otimes \mathbb{C}\left\{\left\{z, z^{-1}\right\}\right\}
$$

equipped with Givental's symplectic form

$$
\begin{aligned}
\Omega: \mathcal{H} \times \mathcal{H} & \longrightarrow \mathbb{C}, \\
(\mathbf{f}(z), \mathbf{g}(z)) & \longmapsto \operatorname{Res}_{z=0}\langle\mathbf{f}(-z), \mathbf{g}(z)\rangle_{H_{X}} d z .
\end{aligned}
$$

It has the standard polarization $\mathcal{H}=\mathcal{H}_{+} \oplus \mathcal{H}_{-}$, where

$$
\mathcal{H}_{+}:=H_{X} \otimes \mathbb{C}\{\{z\}\} \quad \text { and } \quad \mathcal{H}_{-}:=H_{X} \otimes z^{-1} \mathbb{C}\left\{\left\{z^{-1}\right\}\right\} .
$$

The symplectic form $\Omega$ identifies $\mathcal{H}_{-}$with the strong dual of $\mathcal{H}_{+}$and identifies $\mathcal{H}_{+}$with the strong dual of $\mathcal{H}_{-}$. The spaces $\mathcal{H}, \mathcal{H}_{ \pm}$are fully nuclear; $\mathcal{H}_{+}$is Fréchet, and $\mathcal{H}_{-}$is (DF).

Lemma 8.8. Assume that the genus-zero nondescendant potential $F_{X}^{0}$ converges as in Section 2.3. Let $L(t, z)$ be the fundamental solution (37), and let $M(t, z)$ be the inverse fundamental solution (38). Then there exist $\epsilon>0, n \geq 0$, and $R=R\left(\alpha_{1}, \ldots, \alpha_{l}, j_{1}, \ldots, j_{m}\right)>0$ such that for $\left|t^{\alpha}\right|<\epsilon$ and $\left|Q_{i}\right|<\epsilon$, the matrix entries of

$$
\frac{\partial^{l+m} L(t, z)}{\partial t^{\alpha_{1}} \cdots \partial t^{\alpha_{l}} \partial Q_{j_{1}} \cdots \partial Q_{j_{m}}} \text { and } \frac{\partial^{l+m} M(t, z)}{\partial t^{\alpha_{1}} \cdots \partial t^{\alpha_{l}} \partial Q_{j_{1}} \cdots \partial Q_{j_{m}}}
$$

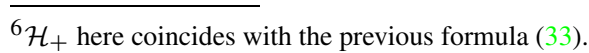


lie in the bounded subset

$$
\left\{\mathbf{a}(z) \in \mathbb{C}\left\{\left\{z^{-1}\right\}\right\}:\|\mathbf{a}(z)\|_{n}<R\right\} .
$$

Proof. Writing $q^{\mathbf{m}}=\left(t^{0}\right)^{m_{0}}\left(Q_{1} e^{t^{1}}\right)^{m_{1}} \cdots\left(Q_{r} e^{t^{r}}\right)^{m_{r}}\left(t^{r+1}\right)^{m_{r+1}} \cdots\left(t^{N}\right)^{m_{N}}$, we can expand the inverse fundamental solution as

$$
M(t, z)=e^{-t / z} \sum_{i=0}^{\infty} \sum_{\mathbf{m}} M_{\mathbf{m}, i} q^{\mathbf{m}_{z}}{ }^{-i}
$$

with $M_{\mathbf{m}, i} \in \operatorname{End}\left(H_{X}\right)$. It was shown ${ }^{7}$ in [25, Lemma 4.1] that $M_{\mathbf{m}, i}$ satisfies

$$
\left\|M_{\mathbf{m}, i}\right\| \leq A C^{|\mathbf{m}|+i} \frac{1}{i !}
$$

for some $A, C>0$. The conclusion about the partial derivatives of $M(t, z)$ follows from this. The same argument as [25, Lemma 4.1] shows the same estimates for the coefficients of $L(t, z)$. This implies the conclusion about the partial derivatives of $L(t, z)$.

Let $\mathbb{C}\{\{z\}\}_{\infty}^{n}$ be the local Banach space of the Fréchet space $\mathbb{C}\{\{z\}\}$ associated to the norm $\|\mathbf{a}(z)\|_{\infty, n}=\sup _{j}\left(\left|a_{j}\right| e^{n j} / j !\right)$, that is,

$$
\mathbb{C}\{\{z\}\}_{\infty}^{n}=\left\{\mathbf{a}(z) \in \mathbb{C} \llbracket z \rrbracket: \sup _{j}\left(\frac{\left|a_{j}\right| e^{n j}}{j !}\right)<\infty\right\} \supset \mathbb{C}\{\{z\}\} .
$$

Then $l_{\infty}^{C}\left(H_{X}\right) \cong H_{X} \otimes \mathbb{C}\{\{z\}\}_{\infty}^{n}$ for $C=e^{n}$ : see (31).

LEMMA 8.9. (1) Let $\mathcal{F}(\mathbf{a})$ be a holomorphic function defined on a neighborhood of the origin of the Fréchet space $\mathbb{C}\{\{z\}\}$. Consider the monomial Taylor expansion

$$
\sum_{\mathbf{m}=\left(m_{0}, m_{1}, m_{2}, \ldots\right)} \frac{1}{m_{0} ! m_{1} ! m_{2} ! \cdots} \frac{\partial^{|\mathbf{m}|} \mathcal{F}}{\partial a_{0}^{m_{0}} \partial a_{1}^{m_{1}} \partial a_{2}^{m_{2}} \cdots}(0) a_{0}^{m_{0}} a_{1}^{m_{1}} a_{2}^{m_{2}} \cdots,
$$

where $\mathbf{m}=\left(m_{0}, m_{1}, m_{2}, \ldots\right)$ is a sequence of nonnegative integers such that $m_{i}=0$ for $i \gg 0$, and $|\mathbf{m}|=\sum_{i} m_{i}$. There exist $n \geq 0$ and $\epsilon>0$ such that the monomial Taylor expansion (44) converges absolutely and uniformly on the $\epsilon$-ball

$$
B_{\epsilon}\left(\mathbb{C}\{\{z\}\}_{\infty}^{n}\right)=\left\{\mathbf{a} \in \mathbb{C}\{\{z\}\}_{\infty}^{n}:\|\mathbf{a}\|_{\infty, n}<\epsilon\right\}
$$

in the Banach space $\mathbb{C}\{\{z\}\}_{\infty}^{n}$ and coincides with $\mathcal{F}(\mathbf{a})$ for $\mathbf{a} \in \mathbb{C}\{\{z\}\} \cap$ $B_{\epsilon}\left(\mathbb{C}\{\{z\}\}_{\infty}^{n}\right)$.

(2) If $\mathcal{F}(\mathbf{a})$ is uniformly continuous with respect to the norm $\|\cdot\|_{\infty, p}$ and holomorphic on the ball $\left\{\mathbf{a}(z) \in \mathbb{C}\{\{z\}\}:\|\mathbf{a}(z)\|_{\infty, p}<\rho\right\}$, then the monomial Taylor expansion (44) converges absolutely and uniformly on the ball $B_{\rho / 3}\left(\mathbb{C}\{\{z\}\}_{\infty}^{p+2}\right)$.

Proof. Let us write the monomial Taylor expansion (44) as

$$
\sum_{\mathbf{m}} \frac{1}{\mathbf{m} !} \mathcal{F}^{(\mathbf{m})}(0) \mathbf{a}^{\mathbf{m}}
$$

\footnotetext{
${ }^{7}$ Note that in [25] $M(t, z)$ is denoted by $L(t,-z)$.
} 
There exist $\eta>0$ and $n \geq 0$ such that $\mathcal{F}$ is holomorphic on

$$
\left\{\mathbf{a} \in \mathbb{C}\{\{z\}\}:\|\mathbf{a}\|_{\infty, n}<2 \eta\right\} .
$$

Decreasing $\eta$ and increasing $n$ if necessary, we can assume that $\mathcal{F}$ is bounded on

$$
\left\{\mathbf{a} \in \mathbb{C}\{\{z\}\}:\|\mathbf{a}\|_{\infty, n} \leq \eta\right\}
$$

since $\mathcal{F}$ is continuous. Set $R_{j}=j ! e^{-n j} \eta$. By the Cauchy integral formula applied to $\mathcal{F}\left(a_{0}, \ldots, a_{l}, 0,0, \ldots\right)$ we obtain for $\mathbf{m}=\left(m_{0}, \ldots, m_{l}, 0,0, \ldots\right)$ :

$$
\begin{aligned}
\left|\frac{1}{\mathbf{m} !} \mathcal{F}^{(\mathbf{m})}(0)\right| & =\left|\frac{1}{(2 \pi i)^{l+1}} \int_{\left|a_{j}\right|=R_{j}, 0 \leq j \leq l} \frac{\mathcal{F}\left(a_{0}, \ldots, a_{l}, 0, \ldots\right)}{a_{0}^{m_{0}+1} \cdots a_{l}^{m_{l}+1}} d a_{0} \cdots d a_{l}\right| \\
& \leq \frac{M e^{n \sum_{j} j m_{j}}}{\eta^{|\mathbf{m}|} \prod_{i}(i !)^{m_{i}}},
\end{aligned}
$$

where $M$ is the supremum of $|\mathcal{F}(\mathbf{a})|$ over $\left\{\|\mathbf{a}\|_{\infty, n} \leq \eta\right\}$. Set $\eta=e \epsilon$. Then if $\|\mathbf{a}\|_{\infty, n+1}<\epsilon$, then we have

$$
\left|\frac{1}{\mathbf{m} !} \mathcal{F}^{(\mathbf{m})}(0) \mathbf{a}^{\mathbf{m}}\right| \leq M e^{-\sum_{i}(i+1) m_{i}} .
$$

The right-hand side is absolutely convergent because

$$
\sum_{\mathbf{m}} e^{-\sum_{i}(i+1) m_{i}}=\prod_{i=0}^{\infty} \frac{1}{1-e^{-i-1}}<\infty .
$$

Hence, the monomial Taylor expansion (44) converges absolutely and uniformly in the ball $B_{\epsilon}\left(\mathbb{C}\{\{z\}\}_{\infty}^{n+1}\right)$. The Taylor series and $\mathcal{F}(\mathbf{a})$ match for $\mathbf{a} \in \mathbb{C}\{\{z\}\}$ with $\|\mathbf{a}\|_{\infty, n+1}<\epsilon$ since both are continuous and they match on the dense subset $\{\mathbf{a} \in$ $\left.\mathbb{C}[z]:\|\mathbf{a}\|_{\infty, n+1}<\epsilon\right\}$. This proves Part (1).

Part (2) can be proved by a small modification of the previous argument. Because $\mathcal{F}$ is uniformly continuous with respect to $\|\cdot\|_{\infty, p}$, it extends uniquely to a $\|\cdot\|_{\infty, p}$-continuous function on the ball

$$
B=\left\{\mathbf{a}(z) \in \mathbb{C}\{\{z\}\}_{\infty}^{p}:\|\mathbf{a}(z)\|_{\infty, p}<\rho\right\} .
$$

In view of the preceding, it suffices to show, under the hypotheses of Part (2), that $\mathcal{F}$ is bounded on

$$
B^{\prime}=\left\{\mathbf{a}(z) \in \mathbb{C}\{\{z\}\}:\|\mathbf{a}(z)\|_{\infty, p+1} \leq e \frac{\rho}{3}\right\} .
$$

Suppose on the contrary that $\mathcal{F}$ is not bounded on $B^{\prime}$. Then there exists a sequence $\left(\mathbf{a}_{l}\right)_{l=1}^{\infty}$ in $B^{\prime}$ such that $\lim _{l \rightarrow \infty}\left|\mathcal{F}\left(\mathbf{a}_{l}\right)\right|=\infty$. Because $\left(\mathbf{a}_{l}\right)_{l=1}^{\infty}$ is bounded in the norm $\|\cdot\|_{\infty, p+1}$, we can find a subsequence $\left(\mathbf{a}_{l_{n}}\right)_{n=1}^{\infty}$ that converges to an element in $B$ in the norm $\|\cdot\|_{\infty, p}$. But $\mathcal{F}$ extends to a continuous function on $B$, so this is a contradiction.

REMARK 8.10. In infinite dimensions there are two different Taylor expansions: monomial expansion as before and the expansion $\sum_{m=0}^{\infty} P_{m}(\mathbf{a}, \ldots, \mathbf{a})$ by $m$-linear forms $P_{m}=\frac{1}{m !} D_{0}^{m} \mathcal{F}$. For a holomorphic function on $\mathbb{C}\{\{z\}\}_{\infty}^{n}$, the monomial expansion does not necessarily converges, whereas $\sum_{m=0}^{\infty} P_{m}(\mathbf{a}, \ldots, \mathbf{a})$ always 
does; see [10] and references therein. On the other hand, Boland and Dineen [4] showed that monomials form an absolute basis of the space of holomorphic functions on the open set $\left\{\mathbf{a} \in \mathbb{C}\{\{z\}\}:\|\mathbf{a}\|_{\infty, n}<\epsilon\right\}$ in $\mathbb{C}\{\{z\}\}$ with respect to a certain topology $\tau_{\omega}$.

\subsection{NF-Convergence of the Genus-Zero Descendant Potential}

In this section we prove Theorem 7.8: that Genus-Zero Convergence (25), which is a convergence assumption on the nondescendant genus-zero potential $F_{X}^{0}$, implies the NF-convergence of the descendant genus-zero potential $\mathcal{F}_{X}^{0}$. The main ingredients are the Nash-Moser inverse function theorem and the reconstruction theorem of Dubrovin [13] and Dijkgraaf and Witten [11], which determines descendant genus-zero invariants from primary genus-zero invariants.

We introduce a sequence of variables $\mathbf{p}=\left(p_{0}, p_{1}, p_{2}, \ldots\right)$ in $H_{X}$ with $p_{i}=$ $\sum_{\alpha=0}^{N} p_{i, \alpha} \phi^{\alpha}$ and a generating function

$$
\mathbf{p}(z)=\sum_{i=0}^{\infty} \sum_{\alpha=0}^{N} p_{i, \alpha} \frac{\phi^{\alpha}}{(-z)^{i+1}}
$$

taking values in $z^{-1} H_{X} \llbracket z^{-1} \rrbracket$. Let $M(t, z)$ denote the inverse fundamental solution (38). Consider the ancestor variable $\mathbf{x}=\left(0, x_{1}, x_{2}, \ldots\right)$ with $x_{0}=0$ and set

$$
\mathbf{q}(z)+\mathbf{p}(z)=M(t, z) \mathbf{x}(z)
$$

(cf. equation (40)), where $\mathbf{q}(z)$ is the nonnegative part, and $\mathbf{p}(z)$ is the strictly negative part. Recall that $\mathbf{x}$ and $\mathbf{y}$ are related by the dilaton shift (41). Because the 0 th ancestor variable $x_{0}=y_{0}$ is now set to equal zero, the map

$$
(t, \mathbf{x}(z)) \mapsto \mathbf{q}(z)=[M(t, z) \mathbf{x}(z)]_{+}
$$

defines an isomorphism between the formal neighborhoods of $\mathbf{y}_{\geq 1}=t=0$ and $\mathbf{t}=0$,

$$
\Lambda \llbracket \mathbf{t} \rrbracket \stackrel{\cong}{\longrightarrow} \Lambda \llbracket \mathbf{y}_{\geq 1} \rrbracket \llbracket t \rrbracket .
$$

(This is clear from equation (42).) Equation (45) determines $\mathbf{p}, \mathbf{q}$ as formal power series in $\mathbf{y}_{\geq 1}$ and $t$. Via that isomorphism, we can regard $p_{i, \alpha}$ as a formal power series in $\mathbf{t}$, that is, as an element of $\Lambda[\mathbf{t} \rrbracket]$.

Theorem 8.11 (Dubrovin [13], Dijkgraaf and Witten [11]). The descendant Gromov-Witten potential $\mathcal{F}_{X}^{0}$ is given by

$$
\mathcal{F}_{X}^{0}=\frac{1}{2} \Omega(\mathbf{p}(z), \mathbf{q}(z))=\frac{1}{2} \sum_{i=0}^{\infty} \sum_{\alpha=0}^{N} p_{i, \alpha} q_{i}^{\alpha} .
$$

Here $\Omega$ is Givental's symplectic form defined in (18).

Proof. Note that the right-hand side converges in the adic topology of $\Lambda \llbracket \mathbf{t} \rrbracket$ because $v\left(q_{i}^{\alpha}\right)=i+1$ for $i \geq 2$. We use a reformulation by Givental [19, Sect. 5], 
proven in Appendix 2 of [9]. The inverse of the coordinate change (46) is given by the fundamental solution $L(t, z)$ in (37) as

$$
0=[L(t, z) \mathbf{q}(z)]_{0}, \quad \mathbf{x}(z)=[L(t, z) \mathbf{q}(z)]_{\geq 1},
$$

where $[\cdots]_{0}$ means the coefficient of $z^{0}$, and $[\cdots]_{\geq 1}$ means the strictly positive truncation of a power series in $z$. The first equation implicitly determines $t$ as a function $t(\mathbf{q})$ of $\mathbf{q}$. We have

$$
\begin{aligned}
\frac{1}{2} \Omega(\mathbf{p}(z), \mathbf{q}(z)) & =\frac{1}{2} \Omega\left([M(t, z) \mathbf{x}(z)]_{-},[M(t, z) \mathbf{x}(z)]_{+}\right) \\
& =\frac{1}{2} \Omega\left(M(t, z)[L(t, z) \mathbf{q}(z)]_{+}, \mathbf{q}(z)\right) \\
& =\frac{1}{2} \Omega\left([L(t, z) \mathbf{q}(z)]_{+}, L(t, z) \mathbf{q}(z)\right)
\end{aligned}
$$

with $t=t(\mathbf{q})$. This coincides with $W_{t}(\mathbf{q}, \mathbf{q}) / 2$ in [19, Prop. 5.3].

Proof of Theorem 7.8. We set:

$$
\begin{aligned}
\mathcal{H}_{\geq 1} & =\left\{\mathbf{x}(z) \in \mathcal{H}_{+}: x_{0}=0\right\}, \\
\Delta_{\epsilon} & =\{a \in \mathbb{C}:|a|<\epsilon\} .
\end{aligned}
$$

By Lemma 8.8 and our convergence assumption for $F_{X}^{0}$, there exist $n \geq 0$ and $\epsilon>0$ such that all the matrix entries of $M(t, z)$ with $\left|t^{\alpha}\right|<\epsilon$ and $\left|Q_{i}\right|<\epsilon$ are bounded with respect to the norm $\|\cdot\|_{n}$. Therefore, if $\mathbf{x} \in \mathcal{H} \geq 1,\left|t^{\alpha}\right|<\epsilon$, and $\left|Q_{i}\right|<\epsilon$, then $(\mathbf{p}, \mathbf{q})$ defined by equation (45) lies in $\mathcal{H}_{-} \times \mathcal{H}_{+}$by Lemma 8.5, and the sum $\sum_{i=0}^{\infty} \sum_{\alpha=0}^{N} p_{i, \alpha} q_{i}^{\alpha}$ converges. Moreover, the map

$$
\begin{aligned}
\mathcal{H}_{\geq 1} \times \Delta_{\epsilon}^{N+1+r} & \rightarrow \mathcal{H}_{-} \times \mathcal{H}_{+}, \\
(\mathbf{x}, t, Q) & \mapsto(\mathbf{p}, \mathbf{q})
\end{aligned}
$$

given by (45) is continuous because

$$
\begin{aligned}
\left\|M(t, z ; Q) \mathbf{x}(z)-M\left(t^{\prime}, z ; Q^{\prime}\right) \mathbf{x}^{\prime}(z)\right\|_{n} \leq & A\left\|(t, Q)-\left(t^{\prime}, Q^{\prime}\right)\right\|\|\mathbf{x}(z)\|_{n+3} \\
& +B\left\|\mathbf{x}(z)-\mathbf{x}^{\prime}(z)\right\|_{n+3}
\end{aligned}
$$

for $n \gg 0$ and some $A, B>0$. This follows from estimate (43) and the uniform estimate of the derivatives of $M(t, z ; Q)$ in Lemma 8.8. The map

$$
(\mathbf{x}, t, Q) \mapsto \frac{1}{2} \Omega(\mathbf{p}(z), \mathbf{q}(z))
$$

is obviously Gâteaux-differentiable and therefore defines a holomorphic function of $(\mathbf{x}, t, Q) \in \mathcal{H}_{\geq 1} \times \Delta_{\epsilon}^{N+1+r}$ (see Remark 7.6). This gives the genus-zero descendant potential $\mathcal{F}_{X}^{0}$ by Theorem 8.11 .

In view of Lemma 8.9, it now suffices to show that the map $(\mathbf{x}, t, Q) \mapsto$ $(\mathbf{q}, Q)$ given by (46) defines a local isomorphism between a neighborhood of $(\mathbf{x}, t, Q)=\left(-\phi_{0} z, 0,0\right)$ in $\mathcal{H}_{\geq 1} \times \mathbb{C}^{N+1+r}$ and a neighborhood of $(\mathbf{q}, Q)=$ $\left(-\phi_{0} z, 0\right)$ in $\mathcal{H}_{+} \times \mathbb{C}^{r}$. We apply the Nash-Moser inverse function theorem [22, Part III, Thm. 1.1.1]. We need to show that there exists a neighborhood $U \subset \mathcal{H}_{\geq 1} \times \mathbb{C}^{N+1+r}$ of $(\mathbf{x}, t, Q)=\left(-\phi_{0} z, 0,0\right)$ such that: 
- the map $(\mathbf{x}, t, Q) \mapsto(\mathbf{q}, Q)$ is smooth tame [22, Part II, §2.1] on $U$;

- the linearized operator $D_{\mathbf{x}, t, Q}(\mathbf{q}, Q)$ is invertible at every $(\mathbf{x}, t, Q) \in U$;

- the inverse $\left(D_{\mathbf{x}, t, Q}(\mathbf{q}, Q)\right)^{-1}$ is continuous and tame as a map $U \times\left(\mathcal{H}_{+} \times\right.$ $\left.\mathbb{C}^{r}\right) \rightarrow \mathcal{H}_{\geq 1} \times \mathbb{C}^{N+1+r}$ (see [22, Part II, Thm. 3.1.1]).

The proof of smoothness is similar to the previous proof of continuity and is omitted. Because $\mathbf{q}$ is linear in $\mathbf{x}$, the smooth-tameness of $(\mathbf{x}, t, Q) \mapsto \mathbf{q}$ follows from the inequality

$$
\left\|\left[\partial_{v_{1}} \cdots \partial_{v_{l}} M(t, z) \mathbf{x}(z)\right]_{+}\right\|_{n} \leq A\|\mathbf{x}\|_{n+3}
$$

for all $(t, Q) \in \Delta_{\epsilon}^{N+1+r}, n \gg 0$, and some $A>0$ ( $A$ can depend on $\left.v_{1}, \ldots, v_{l}\right)$. Here $\partial_{v_{j}}$ denotes the partial derivative along $\Delta_{\epsilon}^{N+1+r}$. Inequality (47) follows directly from estimate (43) and Lemma 8.8.

The linearized operator is given by

$$
\begin{aligned}
& \left(D_{\mathbf{x}, t, Q}(\mathbf{q}, Q)\right)(d \mathbf{x}, d t, d Q) \\
& \quad=\left(\left[M(t, z)\left(-z^{-1} d t * \mathbf{x}+d \mathbf{x}\right)\right]_{+}+\sum_{i=1}^{r} d Q_{i}\left[\frac{\partial M}{\partial Q_{i}}(t, z) \mathbf{x}\right]_{+}, d Q\right),
\end{aligned}
$$

where $*$ denotes the analytic quantum product depending on $(t, Q)$. Equating this with $(d \mathbf{q}, d Q)$, we get

$$
-z^{-1} d t * \mathbf{x}+d \mathbf{x}=\left[L(t, z)\left(d \mathbf{q}-\sum_{i=1}^{r} d Q_{i}\left[\frac{\partial M}{\partial Q_{i}}(t, z) \mathbf{x}\right]_{+}\right)\right]_{+} .
$$

The right-hand side is continuous and tame as a map from $((\mathbf{x}, t, Q),(d \mathbf{q}, d Q)) \in$ $\left(\mathcal{H}_{\geq 1} \times \Delta_{\epsilon}^{N+1+r}\right) \times\left(\mathcal{H}_{+} \times \mathbb{C}^{r}\right)$ to $\mathcal{H}_{+}$for the same reason as before. Equating the left-hand side with $\mathbf{v}=\left(v_{0}, \mathbf{v}_{\geq 1}\right)$ yields

$$
v_{0}=-d t * x_{1}, \quad \mathbf{v}_{\geq 1}=z^{-1} d t * \mathbf{x}_{\geq 2}+d \mathbf{x} .
$$

When $x_{0}$ is sufficiently close to $-\phi_{0}$, the first equation can be inverted, and we obtain the inverse map $((\mathbf{x}, t, Q), \mathbf{v}) \mapsto(d \mathbf{x}, d t)$ given by

$$
d t=-\left(x_{1} *\right)^{-1} v_{0}, \quad d \mathbf{x}=\mathbf{v}_{\geq 1}+z^{-1}\left(\left(x_{1} *\right)^{-1} v_{0}\right) * \mathbf{x}_{\geq 2} .
$$

This map is continuous and tame. Hence, the linearized operator admits a continuous and tame inverse in a neighborhood of $\left(-\phi_{0} z, 0,0\right)$. The Nash-Moser theorem now applies.

An Analytic Version of Theorem 8.11. We saw in Section 8.1 that whenever $\mathcal{F}_{X}^{0}$ converges, we can define the specialization $\mathcal{F}_{X \text {, an }}^{0}$ of $\mathcal{F}_{X}^{0}$ to $Q_{1}=\cdots=Q_{r}=1$. We now show that the Dubrovin-Dijkgraaf-Witten reconstruction theorem (Theorem 8.11) holds for this $\mathcal{F}_{X \text {,an }}^{0}$. Genus Zero Convergence (25) implies that $\left.M(t, z)\right|_{Q_{1}=\cdots=Q_{r}=1}$ depends analytically on $(t, z)$ where $z \in \mathbb{C}^{\times}$and $t$ lies in a neighborhood (4) of the large-radius limit point. Set $\mathcal{H}_{\geq 1}=\left\{\mathbf{x}(z) \in \mathcal{H}_{+}: x_{0}=0\right\}$. We define $\mathbf{q}$ and $\mathbf{p}$ by a formula analogous to (45):

$$
\mathbf{q}(z)+\mathbf{p}(z)=\left.M(t, z)\right|_{Q_{1}=\cdots=Q_{r}=1} \mathbf{x}(z),
$$


where $\mathbf{x}(z) \in \mathcal{H}_{\geq 1}$ and

$$
\mathbf{q}(z)=\left[\left.M(t, z)\right|_{Q_{1}=\cdots=Q_{r}=1} \mathbf{x}(z)\right]_{+} .
$$

Arguing as in the proof of Theorem 7.8 shows that we get a continuous mapping $(t, \mathbf{x}) \mapsto(\mathbf{p}, \mathbf{q}) \in \mathcal{H}_{-} \times \mathcal{H}_{+}$and that the map

$$
\begin{aligned}
\mathcal{H}_{\geq 1} \times H_{X} & \rightarrow \mathcal{H}_{+}, \\
(\mathbf{x}, t) & \mapsto \mathbf{q}(z)
\end{aligned}
$$

gives a local isomorphism between $\mathcal{H}_{\geq 1} \times H_{X}$ and $\mathcal{H}_{+}$for $t$ in a neighborhood (4) of the large-radius limit point and $\mathbf{x}$ in a neighborhood of $-\phi_{0} z$. Therefore, for any chosen point $t \in H_{X} \otimes \mathbb{C}$ in a neighborhood (4) of the large-radius limit point, the sum $\frac{1}{2} \sum_{i=0}^{\infty} \sum_{\alpha=0}^{\infty} p_{i, \alpha} q_{i}^{\alpha}$ can be regarded as a holomorphic function on a neighborhood of $\mathbf{q}(z)=\left[M(t, z)\left(-\phi_{0} z\right)\right]_{+}=t-\phi_{0} z$ in $\mathcal{H}_{+}$.

TheOREM 8.12 (Analytic version of Theorem 8.11). Assume that the genus-zero descendant Gromov-Witten potential $\mathcal{F}_{X}^{0}$ is NF-convergent in the sense of Definition 7.5. Then its specialization $\mathcal{F}_{X \text {, an }}^{0}$ to $Q_{1}=\cdots=Q_{r}=1$ (see (35)) is given by

$$
\mathcal{F}_{X, \text { an }}^{0}=\frac{1}{2} \Omega(\mathbf{p}, \mathbf{q})=\frac{1}{2} \sum_{i=0}^{\infty} \sum_{\alpha=0}^{N} p_{i, \alpha} q_{i}^{\alpha} .
$$

The right-hand side here is, as discussed in the preceding paragraph, a holomorphic function defined on a neighborhood of $\mathbf{q}(z)=t-\phi_{0} z$ in $\mathcal{H}_{+}$, where $t$ is a point in the neighborhood (4) of the large-radius limit point.

Proof. We write the right-hand side as

$$
C^{(0)}(\mathbf{q}):=\frac{1}{2} \Omega(\mathbf{p}, \mathbf{q}) .
$$

The divisor equation shows that (cf. equation (39))

$$
\left.M(t-\delta, z)\right|_{Q_{1}=e^{\delta_{1}}, \ldots, Q_{r}=e^{\delta_{r}}}=\left.e^{\delta / z} M(t, z)\right|_{Q_{1}=\cdots=Q_{r}=1},
$$

where $\delta=\sum_{\alpha=1}^{r} \delta_{\alpha} \phi_{\alpha}$. Therefore, by (48), for $\mathbf{x}=\left(0, x_{1}, x_{2}, \ldots\right)$,

$$
\left.M(t-\delta, z) \mathbf{x}(z)\right|_{Q_{1}=e^{\delta_{1}}, \ldots, Q_{r}=e^{\delta_{r}}}=e^{\delta / z}(\mathbf{p}(z)+\mathbf{q}(z)) .
$$

Assume now that $\Re\left(\delta_{i}\right) \ll 0$ and that $(t-\delta, \mathbf{x})$ is sufficiently close to $\left(0,-\phi_{0} z\right)$. Setting

$$
\begin{aligned}
& \tilde{\mathbf{q}}(z)=\left.[M(t-\delta, z) \mathbf{x}(z)]_{+}\right|_{Q_{1}=e^{\delta_{1}}, \ldots, Q_{r}=e^{\delta_{r}}}=\left[e^{\delta / z} \mathbf{q}(z)\right]_{+}, \\
& \tilde{\mathbf{p}}(z)=\left.[M(t-\delta, z) \mathbf{x}(z)]_{-}\right|_{Q_{1}=e^{\delta_{1}}, \ldots, Q_{r}=e^{\delta_{r}}},
\end{aligned}
$$

we have from the definition of $C^{(0)}$ and the original reconstruction Theorem 8.11 that

$$
\begin{aligned}
C^{(0)}(\mathbf{q}) & =\frac{1}{2} \Omega(\mathbf{p}, \mathbf{q})=\frac{1}{2} \Omega\left(e^{\delta / z} \mathbf{p}, e^{\delta / z} \mathbf{q}\right)=\frac{1}{2} \Omega\left(\tilde{\mathbf{p}}-\left[e^{\delta / z} \mathbf{q}\right]_{-}, \tilde{\mathbf{q}}+\left[e^{\delta / z} \mathbf{q}\right]_{-}\right) \\
& =\frac{1}{2} \Omega(\tilde{\mathbf{p}}, \tilde{\mathbf{q}})-\frac{1}{2} \Omega\left(\left[e^{\delta / z} \mathbf{q}\right]_{-}, \tilde{\mathbf{q}}\right)=\frac{1}{2} \Omega(\tilde{\mathbf{p}}, \tilde{\mathbf{q}})-\frac{1}{2} \Omega\left(e^{\delta / z}\left[e^{-\delta / z} \tilde{\mathbf{q}}\right]_{+}, \tilde{\mathbf{q}}\right)
\end{aligned}
$$




$$
=\mathcal{F}_{X}^{0}\left(\tilde{\mathbf{q}}, e^{\delta_{1}}, \ldots, e^{\delta_{r}}\right)+\frac{1}{2} \Omega\left(e^{-\delta / z} \tilde{\mathbf{q}},\left[e^{-\delta / z} \tilde{\mathbf{q}}\right]_{+}\right) .
$$

Applying (36), we see that the right-hand side coincides with $\mathcal{F}_{X \text {,an }}^{0}(\mathbf{q})$.

\subsection{The Proof of Theorem 7.9}

The genus-zero ancestor potential $\overline{\mathcal{F}}_{X}^{0}$ contains as a subseries

$$
\sum_{d \in \operatorname{NE}(X)} \sum_{n=0}^{\infty} \sum_{0 \leq \alpha, \beta, \gamma \leq N} \frac{Q^{d}}{3 ! n !}\langle y_{0}^{\alpha} \phi_{\alpha}, y_{0}^{\beta} \phi_{\beta}, y_{0}^{\gamma} \phi_{\gamma} ; \overbrace{t, \ldots, t}^{n}\rangle_{0,3+n, d}^{X},
$$

and our convergence assumption implies that the coefficient of $y_{0}^{\alpha} y_{0}^{\beta} y_{0}^{\gamma}$ converges as a power series in $t$ and $Q$. This shows that all third derivatives of the nondescendant genus-zero potential $F_{X}^{0}$ are convergent and thus that $F_{X}^{0}$ itself is convergent. Theorem 7.8 then implies that the genus-zero descendant potential $\mathcal{F}_{X}^{0}$ is NF-convergent.

A similar argument shows that the genus-one nondescendant potential $F_{X}^{1}(t)$ converges. All derivatives of $F_{X}^{1}(t)$ appear as subseries of $\overline{\mathcal{F}}_{t}^{1}$ and hence are convergent. Thus, $F_{X}^{1}(t)$ is also convergent. Now the ancestor-descendant relation (Theorem 8.3) leads to the NF-convergence of higher-genus descendant potentials. Under our convergence assumptions, the ancestor potential $\overline{\mathcal{F}}_{t}^{g}(\mathbf{x})$ with $x_{0}$ set to equal zero depends:

- analytically on $t^{i}, Q_{i}$ in a region (17), for some $\epsilon>0$,

- rationally on $x_{1}$, and

- polynomially on $x_{2}, x_{3}, \ldots$.

In particular, it is holomorphic in a small neighborhood of $(\mathbf{x}, t, Q)=\left(-\phi_{0} z, 0,0\right)$ in the Fréchet space $\mathcal{H}_{\geq 1} \times \mathbb{C}^{N+1+r}$; moreover, for every $n \geq 0$, it is uniformly continuous with respect to the norm $\|\cdot\|_{n}$ in a $\|\cdot\|_{n}$-neighborhood of $\left(-\phi_{0} z, 0,0\right)$. On the other hand, in the proof (Section 8.5) of Theorem 7.8, we used the Nash-Moser inverse function theorem to show that the coordinate change $(\mathbf{x}, t, Q) \mapsto(\mathbf{q}, Q)$ defined by (46) is an isomorphism between a neighborhood of $\left(-\phi_{0} z, 0,0\right)$ in $\mathcal{H}_{\geq 1} \times \mathbb{C}^{N+1+r}$ and a neighborhood of $\left(-\phi_{0} z, 0\right)$ in $\mathcal{H}_{+} \times \mathbb{C}^{r}$. The Nash-Moser theorem [22, Part III, Thm. 1.1.1] moreover asserts that the inverse map $(\mathbf{q}, Q) \mapsto(\mathbf{x}, t, Q)$ is smooth tame. Therefore, there exist $m \geq n \geq 0$ such that the inverse map is defined on a $\|\cdot\|_{m}$-neighborhood of $\left(-z \phi_{0}, 0\right)$ and is $\left(\|\cdot\|_{m},\|\cdot\|_{n}\right)$-Lipschitz continuous ${ }^{8}$ there, that is, there exists $C>0$ such that

$$
\left\|\left(\mathbf{x}^{\prime}, t^{\prime}, Q^{\prime}\right)-(\mathbf{x}, t, Q)\right\|_{n} \leq C\left\|\left(\mathbf{q}^{\prime}, Q^{\prime}\right)-(\mathbf{q}, Q)\right\|_{m}
$$

for all $(\mathbf{q}, Q)$ and $\left(\mathbf{q}^{\prime}, Q^{\prime}\right)$ in a $\|\cdot\|_{m}$-neighborhood of $\left(-z \phi_{0}, 0\right)$, where $\left(\mathbf{x}^{\prime}, t^{\prime}, Q^{\prime}\right)$ and $(\mathbf{x}, t, Q)$ are the inverse images of $\left(\mathbf{q}^{\prime}, Q^{\prime}\right)$ and $(\mathbf{q}, Q)$, respectively. By the ancestor-descendant relation (Theorem 8.3) the descendant potentials $\mathcal{F}^{g}(\mathbf{q})$, $g \geq 1$ are the pull-backs of the ancestor potentials $\overline{\mathcal{F}}_{t}^{g}(\mathbf{x})+\delta_{g, 1} F^{1}(t)$ under the inverse map $(\mathbf{q}, Q) \mapsto(\mathbf{x}, t, Q)$. Therefore, $\mathcal{F}^{g}(\mathbf{q}), g \geq 1$, are holomorphic and

\footnotetext{
${ }^{8}$ To show this, we apply [22, Part II, Lemma 2.1.7] to the derivative of the inverse map.
} 
uniformly continuous with respect to $\|\cdot\|_{m}$ on a common (i.e., independent of $g)\|\cdot\|_{m}$-neighborhood of $\left(-z \phi_{0}, 0\right)$ in $\mathcal{H}_{+} \times \mathbb{C}^{r}$. Because $\|\cdot\|_{m} \leq C\|\cdot\|_{\infty, m+1}$ for some $C>0$, the same holds for the norm $\|\cdot\|_{\infty, m+1}$. By Lemma 8.9 (2), the monomial Taylor expansions of $\mathcal{F}^{g}(\mathbf{q}), g \geq 1$, converge uniformly and absolutely on a common Banach ball for the norm $\|\cdot\|_{\infty, m+3}$. The conclusion follows.

\subsection{The Proof of Theorem 7.3}

By Theorem 7.9 the total descendant potential $\mathcal{Z}_{X}$ is convergent in the sense of Definition 7.5. In particular, the genus-zero nondescendant potential $F_{X}^{0}$ is convergent. In this case the coordinate change (40) appearing in the ancestor-descendant relation (Theorem 8.3) is an isomorphism between a neighborhood of $(\mathbf{x}, t, Q)=$ $\left(-\phi_{0} z, 0,0\right)$ in $\mathcal{H}_{+} \times \mathbb{C}^{N+1+r}$ and a neighborhood of $(\mathbf{q}, t, Q)=\left(-\phi_{0} z, 0,0\right)$ in $\mathcal{H}_{+} \times \mathbb{C}^{N+1+r}$. (See equation (33) for $\mathcal{H}_{+}$.) This follows from the Nash-Moser inverse function theorem using almost the same argument as in the proof of Theorem 7.8. Therefore, the genus- $g$ ancestor potentials $\overline{\mathcal{F}}_{t}^{g}(\mathbf{x})$ for all $g \geq 1$ are holomorphic on a common neighborhood of $(\mathbf{x}, t, Q)=\left(-\phi_{0} z, 0,0\right)$ and thus are NFconvergent by Lemma 8.9. (We will prove in the next paragraph that the constants $C$ and $\epsilon$ defining the radius of NF-convergence here can be taken to be independent of $g$.) At genus zero, the ancestor-descendant relation takes the form [19, Prop. 5.3]

$$
\mathcal{F}^{0}(\mathbf{q})=\overline{\mathcal{F}}_{t}^{0}(\mathbf{x})+\frac{1}{2} W_{t}(\mathbf{q}, \mathbf{q})
$$

where the quadratic form $W_{t}(\mathbf{q}, \mathbf{q})$ appeared in the proof of Theorem 8.11; it is convergent and holomorphic if $\mathbf{q}$ lies in $\mathcal{H}_{+}$and $\left|t^{\alpha}\right|,\left|Q_{i}\right|$ are sufficiently small (cf. the proof of Theorem 7.8). The NF-convergence of $\overline{\mathcal{F}}_{t}^{0}(\mathbf{x})$ follows.

For the uniformity of the constants $C, \epsilon>0$, we use an argument similar to the proof (Section 8.6) of Theorem 7.9. We know that $\mathcal{F}_{g}(\mathbf{q})$ is uniformly continuous for $\|\cdot\|_{n}$ on a common (i.e., independent of $g \geq 1$ ) $\|\cdot\|_{n}$-neighborhood of $(\mathbf{q}, t, Q)=\left(-z \phi_{0}, 0,0\right)$ for some $n \geq 0$ and that the map $(\mathbf{x}, t, Q) \mapsto(\mathbf{q}, t, Q)$ is smooth tame; thus, the pull-backs of $\mathcal{F}^{g}(\mathbf{q})$ by $(\mathbf{x}, t, Q) \mapsto(\mathbf{q}, t, Q)$ are uniformly continuous for $\|\cdot\|_{m}$ on a common $\|\cdot\|_{m}$-neighborhood of $(\mathbf{x}, t, Q)=$ $\left(-\phi_{0} z, 0,0\right)$ for some $m \geq n$. Then we apply part 2 of Lemma 8.9 to find that the monomial Taylor expansion of $\overline{\mathcal{F}}_{t}^{g}(\mathbf{x})$ converges uniformly and absolutely on a common $\|\cdot\|_{\infty, m+3}$-neighborhood of $\left(-\phi_{0} z, 0,0\right)$.

\section{Negative Line Bundles Over Compact Toric Varieties}

We now prove Corollary 1.3. Let $Y$ be a compact toric variety, and let $X$ be the total space of a direct sum $E=\bigoplus_{j=1}^{j=r} E_{j}$ of line bundles $E_{j}$ over $Y$ such that $c_{1}\left(E_{j}\right) \cdot d<0$ whenever $d$ is the degree of a holomorphic curve in $Y$. In what follows, we take $r=1$, leaving the proof of the general case (which is very similar) to the reader. Let $\bar{X}$ denote the projectivization $\bar{X}=\mathbb{P}(E \oplus \mathbb{C})$, and let $X_{\infty} \subset \bar{X}$ denote the infinity section. The inclusion $i: X \rightarrow \bar{X}$ induces a map

$$
i_{\star}: H_{2}(X ; \mathbb{Z}) \rightarrow H_{2}(\bar{X}, \mathbb{Z}),
$$


and $e \in H_{2}(X, \mathbb{Z})$ satisfies $e=i_{\star}(d)$ if and only if $e \cdot\left[X_{\infty}\right]=0$. Thus, since $E \rightarrow X$ is negative, any nonconstant holomorphic curve in $\bar{X}$ in the class $i_{\star}(d)$ lies entirely inside the zero section of $\bar{X}$. It follows that

$$
\left\langle a_{1} \psi_{1}^{i_{1}}, \ldots, a_{n} \psi_{n}^{i_{n}}\right\rangle_{g, n, i_{\star}(d)}^{\bar{X}}=\left\langle i^{\star}\left(a_{1}\right) \psi_{1}^{i_{1}}, \ldots, i^{\star}\left(a_{n}\right) \psi_{n}^{i_{n}}\right\rangle_{g, n, d}^{X}, \quad d \neq 0,
$$

where the right-hand side is defined as a local Gromov-Witten invariant [6], and hence that the total descendant potential $\mathcal{Z}_{X}$ occurs as a subseries of the total descendant potential $\mathcal{Z}_{\bar{X}}$. (Note that the degree-zero Gromov-Witten invariants of $X$ are not defined because the relevant moduli spaces of stable maps are not compact, and hence degree-zero terms are omitted in the definition of $\mathcal{Z}_{X}$.) Corollary 1.2 implies that the total descendant potential $\mathcal{Z}_{\bar{X}}$ is NF-convergent in the sense of Definition 7.5, and it follows that $\mathcal{Z}_{X}$ is NF-convergent too. The proof of Corollary 1.3 is complete.

Proposition 9.1. Let $X$ be a compact toric variety or a complete flag variety. The total ancestor potential $\mathcal{A}_{X}$ is convergent in the sense of Definition 3.13 and is $N F$-convergent in the sense of Definition 7.1.

Proof. Combine the argument in the proof of Corollary 1.2 with Theorem 1.4.

Proposition 9.2. Let $Y$ be a compact toric variety, and let $X$ be the total space of a direct sum $E=\bigoplus_{j=1}^{j=r} E_{j}$ of line bundles $E_{j}$ over $Y$ such that $c_{1}\left(E_{j}\right) \cdot d<0$ whenever $d$ is the degree of a holomorphic curve in $Y$. The total ancestor potential $\mathcal{A}_{X}$ is convergent in the sense of Definition 3.13 and is NF-convergent in the sense of Definition 7.1.

Proof. Argue as in the proof of Corollary 1.3, but use Proposition 9.1 in place of Corollary 1.2.

AcKnowledgments. We are grateful to Hsian-Hua Tseng for very useful discussions on Givental quantization. The definition of Fock spaces for ancestor potentials was originally worked out in another joint project with him, and we thank him for allowing us to present this formulation here. We thank Yongbin Ruan and Yefeng Shen for giving us a strong motivation for writing up this paper. TC thanks Konstanze Rietsch for a useful conversation about flag varieties.

\section{References}

[1] D. Abramovich, T. Graber, and A. Vistoli, Gromov-Witten theory of DeligneMumford stacks, Amer. J. Math. 130 (2008), no. 5, 1337-1398.

[2] F. Aroca, Puiseux parametric equations of analytic sets, Proc. Amer. Math. Soc. 132 (2004), no. 10, 3035-3045 (electronic).

[3] K. Behrend and B. Fantechi, The intrinsic normal cone, Invent. Math. 128 (1997), no. $1,45-88$.

[4] P. J. Boland and S. Dineen, Holomorphic functions on fully nuclear spaces, Bull. Soc. Math. France 106 (1978), no. 3, 311-336, English, with French summary. 
[5] S. B. Chae, Holomorphy and calculus in normed spaces, Monographs and Textbooks in Pure and Applied Mathematics, 92, Marcel Dekker Inc., New York, 1985, with an appendix by Angus E. Taylor.

[6] T.-M. Chiang, A. Klemm, S.-T. Yau, and E. Zaslow, Local mirror symmetry: calculations and interpretations, Adv. Theor. Math. Phys. 3 (1999), no. 3, 495-565.

[7] T. Coates, A. Corti, H. Iritani, and H.-H. Tseng, A mirror theorem for toric stacks, arXiv:1310.4163, Compos. Math. (to appear). DOI:10.1112/S0010437X15007356.

[8] T. Coates, A. Corti, Y.-P. Lee, and H.-H. Tseng, The quantum orbifold cohomology of weighted projective spaces, Acta Math. 202 (2009), no. 2, 139-193.

[9] T. Coates and A. Givental, Quantum Riemann-Roch, Lefschetz and Serre, Ann. of Math. (2) 165 (2007), no. 1, 15-53.

[10] A. Defant, M. Maestre, and C. Prengel, Domains of convergence for monomial expansions of holomorphic functions in infinitely many variables, J. Reine Angew. Math. 634 (2009), 13-49.

[11] R. Dijkgraaf and E. Witten, Mean field theory, topological field theory, and multimatrix models, Nuclear Phys. B 342 (1990), no. 3, 486-522.

[12] S. Dineen, Complex analysis on infinite-dimensional spaces, Springer Monogr. Math., Springer-Verlag London Ltd., London, 1999.

[13] B. Dubrovin, Geometry of 2D topological field theories, Integrable systems and quantum groups (Montecatini Terme, 1993), Lecture Notes in Math., 1620, pp. 120-348, Springer, Berlin, 1996.

[14] _ Painlevé transcendents in two-dimensional topological field theory, The Painlevé property, CRM Ser. Math. Phys., pp. 287-412, Springer, New York, 1999.

[15] H. Fan, T. Jarvis, and Y. Ruan, The Witten equation, mirror symmetry and quantum singularity theory, Ann. of Math. (2) 178 (2013), 1-106.

[16] A. B. Givental, Stationary phase integrals, quantum Toda lattices, flag manifolds and the mirror conjecture, Topics in singularity theory, Amer. Math. Soc. Transl. Ser. 2, 180, pp. 103-115, Amer. Math. Soc., Providence, RI, 1997.

[17] _ A mirror theorem for toric complete intersections, Topological field theory, primitive forms and related topics (Kyoto, 1996), Progr. Math., 160, pp. 141-175, Birkhäuser Boston, Boston, MA, 1998.

[18] _ Semisimple Frobenius structures at higher genus, Int. Math. Res. Not. IMRN 23 (2001), 1265-1286.

[19] _ Gromov-Witten invariants and quantization of quadratic Hamiltonians, Mosc. Math. J. 1 (2001), no. 4, 551-568, 645. English, with English and Russian summaries, Dedicated to the memory of I. G. Petrovskii on the occasion of his 100th anniversary.

[20] _,$A_{n-1}$ singularities and $n K d V$ hierarchies, Mosc. Math. J. 3 (2003), no. 2, 475-505, 743. English, with English and Russian summaries, Dedicated to Vladimir I. Arnold on the occasion of his 65 th birthday.

[21] _ Symplectic geometry of Frobenius structures, Frobenius manifolds, Aspects Math., E36, pp. 91-112, Vieweg, Wiesbaden, 2004.

[22] R. S. Hamilton, The inverse function theorem of Nash and Moser, Bull. Amer. Math. Soc. (N.S.) 7 (1982), no. 1, 65-222.

[23] C. Hertling, Y. I. Manin, and C. Teleman, An update on semisimple quantum cohomology and F-manifolds, Tr. Mat. Inst. Steklova 264 (2009), no. Mnogomernaya Algebraicheskaya Geometriya 69-76, English transl., Proc. Steklov Inst. Math. 264 (2009), no. 1, 62-69.

[24] K. Hori and C. Vafa, Mirror symmetry, 2000, arXiv:hep-th/0002222. 
[25] H. Iritani, Convergence of quantum cohomology by quantum Lefschetz, J. Reine Angew. Math. 610 (2007), 29-69.

[26] An integral structure in quantum cohomology and mirror symmetry for toric orbifolds, Adv. Math. 222 (2009), no. 3, 1016-1079.

[27] , 2004, unpublished.

[28] B. Kim, Quantum cohomology of flag manifolds $G / B$ and quantum Toda lattices, Ann. of Math. (2) 149 (1999), no. 1, 129-148.

[29] M. Kontsevich and Y. Manin, Gromov-Witten classes, quantum cohomology, and enumerative geometry, Comm. Math. Phys. 164 (1994), no. 3, 525-562.

[30] , Relations between the correlators of the topological sigma-model coupled to gravity, Comm. Math. Phys. 196 (1998), no. 2, 385-398.

[31] B. Kostant, Flag manifold quantum cohomology, the Toda lattice, and the representation with highest weight $\rho$, Selecta Math. (N.S.) 2 (1996), no. 1, 43-91.

[32] J. Li and G. Tian, Virtual moduli cycles and Gromov-Witten invariants of algebraic varieties, J. Amer. Math. Soc. 11 (1998), no. 1, 119-174.

[33] Y. I. Manin, Frobenius manifolds, quantum cohomology, and moduli spaces, Amer. Math. Soc. Colloq. Publ., 47, American Mathematical Society, Providence, RI, 1999.

[34] T. E. Milanov and H.-H. Tseng, Equivariant orbifold structures on the projective line and integrable hierarchies, Adv. Math. 226 (2011), no. 1, 641-672.

[35] T. Reichelt, A construction of Frobenius manifolds with logarithmic poles and applications, Comm. Math. Phys. 287 (2009), no. 3, 1145-1187.

[36] C. Teleman, The structure of 2D semi-simple field theories, Invent. Math. 188 (2012), 525-588.

[37] E. Witten, Algebraic geometry associated with matrix models of two-dimensional gravity, Topological methods in modern mathematics (Stony Brook, NY, 1991), pp. 235-269, Publish or Perish, Houston, TX, 1993.

Department of Mathematics

Imperial College London

180 Queen's Gate

London SW7 2AZ

United Kingdom

t.coates@imperial.ac.uk
Department of Mathematics

Graduate School of Science

Kyoto University

Kitashirakawa-Oiwake-cho

Sakyo-ku, Kyoto, 606-8502

Japan

iritani@math.kyoto-u.ac.jp 